\title{
RESIDUE CURRENTS \\ OF THE BOCHNER-MARTINELLI TYPE
}

\author{
Mikael Passare, August Tsikh and Alain Yger
}

\begin{abstract}
Our objective is to construct residue currents from Bochner-Martinelli type kernels; the computations hold in the non complete intersection case and provide a new and more direct approach of the residue of Coleff-Herrera in the complete intersection case; computations involve crucial relations with toroidal varieties and multivariate integrals of the Mellin-Barnes type.
\end{abstract}

\section{Introduction}

Of the great number of integral representation formulas for holomorphic functions in several variables, there are two that are particularly simple and useful, namely those given by the Cauchy kernel and by the Bochner-Martinelli kernel. It is well known, see [18], that these kernels correspond to each other via the Dolbeault isomorphism. Moreover, it is an elementary observation that the Bochner-Martinelli representation formula can be obtained by averaging the Cauchy formula over a simplex. More precisely, taking the mean value over the simplex

$$
\Sigma_{p}(\eta)=\left\{s \in \mathbf{R}_{+}^{n} ; s_{1}+\cdots+s_{p}=\eta\right\}
$$

of both sides in the Cauchy formula

$$
h(0)=\frac{1}{(2 \pi i)^{p}} \int_{\left|w_{j}\right|^{2}=s_{j}} \frac{h(w) d w_{1} \wedge \cdots \wedge d w_{p}}{w_{1} \cdots w_{p}}
$$

1991 Mathematics Subject Classification. 32A27, 32C30, 32S40, 33C70. 
one arrives at the Bochner-Martinelli formula

$$
h(0)=c_{p} \int_{\|w\|^{2}=\eta} h(w) \Omega(w) \wedge d w_{1} \wedge \cdots \wedge d w_{p},
$$

where $c_{p}=(-1)^{p(p-1) / 2}(p-1) ! /(2 \pi i)^{p}$ is a constant depending only on the number of variables, and the kernel $\Omega$ is given by

$$
\Omega(w)=\frac{1}{\|w\|^{2 p}} \sum_{k=1}^{p}(-1)^{k-1} \bar{w}_{k} d \bar{w}_{1} \wedge \cdots \widehat{d \bar{w}_{k}} \cdots \wedge d \bar{w}_{p} .
$$

The simplicity of the Cauchy kernel makes it a natural candidate in the definition of multidimensional residues. For instance, there is an elegant integral interpretation of the Grothendieck residue based on this kernel, see [19]. In 1978 the Cauchy kernel was used by Coleff and Herrera [14] in their definition of residue currents, which goes as follows: Let $f_{1}, \ldots, f_{p}$ be a system of $p$ holomorphic functions in some domain $V \subset \mathbf{C}^{n}$. For every smooth, compactly supported test form $\varphi \in \mathcal{D}^{n, n-p}(V)$ one considers the integral

$$
I(\varepsilon)=I(\varepsilon ; \varphi)=\frac{1}{(2 \pi i)^{p}} \int_{\left|f_{j}\right|^{2}=\varepsilon_{j}} \frac{\varphi}{f_{1} \cdots f_{p}},
$$

where the real-analytic chain $\left\{\left|f_{1}\right|^{2}=\varepsilon_{1}, \ldots,\left|f_{p}\right|^{2}=\varepsilon_{p}\right\}$ is oriented as the distinguished boundary of the corresponding polyhedron. It is easy to see that, when the common zero set $f^{-1}(0)$ of the system $f=$ $\left(f_{1}, \ldots, f_{p}\right)$ has codimension less than $p$ (that is, when $f$ is not a complete intersection), then the function $I(\varepsilon)$ given by (1.1) does not have a limit as $\varepsilon \rightarrow 0$. However, Coleff and Herrera showed that this limit does exist if one lets $\varepsilon$ approach the origin along a special path $\varepsilon(\delta)=$ $\left(\varepsilon_{1}(\delta), \ldots, \varepsilon_{p}(\delta)\right)$, a so-called admissible trajectory, for which each coordinate tends to zero quicker than any power of the subsequent coordinate. In the case of a complete intersection this limit is independent of the ordering of the functions, and it seemed reasonable to expect, in this case, the existence of an unconditional limit of the function $I(\varepsilon)$ at the origin. This turned out not to be the case, and the counterexamples of [25] and [12] show that the behaviour of the integral (1.1) near $\varepsilon=0$ can be quite intricate. We have therefore found it natural to consider the residue current, associated with the mapping $f: V \rightarrow \mathbf{C}^{p}$, as a limit of certain averages of the residue function $I(\varepsilon)$. 
The aim of the present paper is to study residue currents of the Bochner-Martinelli type, which may be viewed as limits of mean values of $I(\varepsilon)$ over the simplex $\Sigma_{p}(\eta)$ and which can in fact be written as

$$
T_{f}(\varphi)=\lim _{\eta \rightarrow 0} c_{p} \int_{\|f\|^{2}=\eta} \Omega(f) \wedge \varphi .
$$

In particular, our Theorem 1.1 says that such a limit always exists and defines a $(0, p)$-current $T_{f}$, which annihilates the integral closure of the $p$-th power of the ideal generated by $f_{1}, \ldots, f_{p}$ in the space of holomorphic functions in $V$, and which also annihilates the conjugate of any function from the radical of this ideal. In the complete intersection case $T_{f}$ coincides with the Coleff-Herrera current, see Theorem 4.1, and with the currents considered in the papers $[\mathbf{2 3}],[\mathbf{5}],[\mathbf{2 4}]$, so there is the natural notation

$$
T_{f}=\bar{\partial} \frac{1}{f_{1}} \wedge \cdots \wedge \bar{\partial} \frac{1}{f_{p}} .
$$

As a consequence we obtain in Theorem 2.1 the alternative representation

$$
T_{f}(\varphi)=\lim _{\tau \rightarrow 0} p c_{p} \int_{V} \frac{\tau \overline{\partial f} \wedge \varphi}{\left(\|f\|^{2}+\tau\right)^{p+1}},
$$

where $\overline{\partial f}=\overline{\partial f_{1}} \wedge \cdots \wedge \overline{\partial f_{p}}$, for the current $\bar{\partial}\left(1 / f_{1}\right) \wedge \cdots \wedge \bar{\partial}\left(1 / f_{p}\right)$. This latter limit agrees with a more classical approach to particularly simple residue currents (with measure coefficients), which was used in [3] for obtaining interpolation and division formulas.

We feel that our results are of a certain interest already in the case of a complete intersection $f$. Indeed, a big draw-back in the theory of residue currents has always been the difficulty (for $p>1$ ) in giving a concise definition of them, and the above limits (1.2) and (1.3) certainly provide much more appealing definitions of $T_{f}$ than the previously existing ones. (We must admit though that we had to do some work in order to prove their equivalence.)

We shall however not restrict ourselves to the complete intersection case. This is partly because in our existence proof we do not need this assumption, but more importantly since there is already some recent work (see for example [30], [9]), where some questions related to residue theory in the non-complete intersection case are studied.

Here is the exact formulation of our main result: 
Theorem 1.1. Let $f_{1}, \ldots, f_{m}$, be $m$ holomorphic functions defined in some open set $V$ of $\mathbf{C}^{n}$. Then, for any ordered subset $\mathcal{I} \subset\{1, \ldots, m\}$ of cardinality $p \leq \min (m, n)$, and for any test form $\varphi \in \mathcal{D}^{n, n-p}(V)$, the limit

$$
\lim _{\eta \rightarrow 0} \frac{c_{p}}{\eta^{p}} \int_{\left\{\|f\|^{2}=\eta\right\}}\left(\sum_{k=1}^{p}(-1)^{k-1} \overline{f_{i_{k}}} \bigwedge_{l \neq k} \overline{d f_{i_{l}}}\right) \wedge \varphi
$$

exists and defines the action of $a(0, p)$ current $T_{f, \mathcal{I}}$ with the following vanishing properties:

(i) $\bar{h} T_{f, \mathcal{I}}=0$ for any $h \in \mathcal{O}(V)$ which vanishes on the common zero set $\left\{f_{1}=\cdots=f_{m}=0\right\}$;

(ii) $h T_{f, \mathcal{I}}=0$ for any $h \in \mathcal{O}(V)$ which is locally in the integral closure of the ideal $\left(f_{1}, \ldots, f_{m}\right)^{p}$;

(iii) $T_{f, \mathcal{I}}=0$ if $p<\operatorname{codim}\left\{f_{1}=\cdots=f_{m}=0\right\}$.

Moreover, $T_{f, \mathcal{I}}$ depends in an alternating way on the ordering of the elements in $\mathcal{I}$.

Since the currents we introduce here are similar to those introduced in [15, Section 5], it seems reasonable to expect that the constructions we propose in this paper might give some further insight regarding explicit formulations of the Ehrenpreis-Palamodov fundamental principle in the non complete intersection case (in the spirit of the formulation in [13]).

Finally, we will also explain in our paper how explicit computations involving Bochner-Martinelli currents (in the case of normal crossings, when the $f_{j}$ are monomials) provide interesting connections with multidimensional Mellin-Barnes integrals (see $[\mathbf{2 7}]$ ).

\section{Residue currents of the Bochner-Martinelli type}

In this section we give a proof of Theorem 1.1. First a piece of notation: Throughout this paper $c_{p}$ will denote the numerical constant $(-1)^{p(p-1) / 2}(p-1) ! /(2 \pi i)^{p}$. Consider an open set $V$ in $\mathbf{C}^{n}$ and let $f_{1}, \ldots, f_{m}$ be elements in the algebra $\mathcal{O}(V)$ of holomorphic functions in $V$. It follows from Sard's theorem that there is a negligible set $E_{f}$, such that for each $\eta \in \mathbf{R}_{+} \backslash E_{f}$ the equation

$$
\|f(\zeta)\|^{2}:=\sum_{k=1}^{m}\left|f_{k}(\zeta)\right|^{2}=\eta
$$


defines a smooth real hypersurface in $V$, which inherits the standard orientation of $V \subset \mathbf{C}^{n}$. We denote by $\Gamma_{f}(\eta)$ the corresponding real analytic $(2 n-1)$-chain. Let further $\mathcal{I}=\left\{i_{1}, \ldots, i_{p}\right\}$ be an arbitrary ordered subset of $\{1, \ldots, m\}$, whose number of elements $p$ is at most $\min (n, m)$. For any test form $\varphi \in \mathcal{D}^{n, n-p}(V)$ and for each $\eta \in \mathbf{R}_{+} \backslash E_{f}$, we then write

$$
J_{f, \mathcal{I}}(\varphi, \eta):=\frac{c_{p}}{\eta^{p}} \int_{\left\{\|f\|^{2}=\eta\right\}}\left(\sum_{k=1}^{p}(-1)^{k-1} \overline{f_{i_{k}}} \bigwedge_{l \neq k} \overline{d f_{i_{l}}}\right) \wedge \varphi .
$$

It follows from the co-area formula ([16, Theorem 3.2.11, p. 248]) that the almost everywhere defined map

$$
\mathbf{R}_{+} \ni \eta \mapsto J_{f, \mathcal{I}}(\varphi, \eta)
$$

defines a compactly supported element in the weighted space $L^{1}\left(\mathbf{R}_{+}, t^{p} d t\right)$. Therefore, its Mellin transform

$$
\mathbf{C} \ni \lambda \mapsto M_{f, \mathcal{I}}(\varphi, \lambda):=\lambda \int_{0}^{\infty} J_{f, \mathcal{I}}(\varphi, \eta) \eta^{\lambda-1} d \eta
$$

is a holomorphic function in the half-plane $\operatorname{Re} \lambda>p$.

Lemma 2.1. For $\operatorname{Re} \lambda>p$, the above Mellin transform may be represented as

$$
\begin{aligned}
& M_{f, \mathcal{I}}(\varphi, \lambda) \\
& \quad=c_{p} \int_{V} \lambda\|f\|^{2(\lambda-p-1)} \bar{\partial}\|f\|^{2} \wedge\left(\sum_{k=1}^{p}(-1)^{k-1} \overline{f_{i_{k}}} \bigwedge_{l \neq k} \overline{d f_{i_{l}}}\right) \wedge \varphi .
\end{aligned}
$$

Proof: Let $E_{f}(\varphi)$ denote the set of critical values for the mapping $\zeta \rightarrow$ $\|f(\zeta)\|^{2}$ restricted to $\operatorname{Supp} \varphi$. It is a closed subset of $\mathbf{R}_{+}$contained in the negligible set $E_{f}$. If $] \alpha, \beta$ is any open interval in $\mathbf{R}_{+} \backslash E_{f}(\varphi)$, we get from Fubini's theorem that, for any $\lambda \in \mathbf{C}$,

$$
\begin{aligned}
& \int_{\alpha}^{\beta} \lambda J_{f, \mathcal{I}}(\varphi, \eta) \eta^{\lambda-1} d \eta \\
& \quad=\int_{V_{\alpha \beta}} \lambda\|f\|^{2(\lambda-p-1)} \bar{\partial}\|f\|^{2} \wedge\left(\sum_{k=1}^{p}(-1)^{k-1} \overline{f_{i_{k}}} \bigwedge_{l \neq k} \overline{d f_{i_{l}}}\right) \wedge \varphi
\end{aligned}
$$


where $V_{\alpha \beta}$ denotes the set $\left\{\zeta \in V ; \alpha<\|f\|^{2}<\beta\right\}$. The set $\mathbf{R}_{+} \backslash E_{f}(\varphi)$ is a countable union of such disjoint intervals $] \alpha, \beta$ [, so it follows from Lebesgue's theorem and from the co-aerea formula that the equality $(2.2)$ holds for all $\lambda$ with $\operatorname{Re} \lambda>p$.

Our second lemma gives the existence of a meromorphic continuation of the Mellin transform $M_{f, \mathcal{I}}$ which is in fact holomorphic across the imaginary axis. Its value at the origin is of particular interest to us.

Lemma 2.2. The function $\lambda \mapsto M_{f, \mathcal{I}}(\varphi, \lambda)$ can be meromorphically continued to the whole complex plane, and the poles of the extended function are strictly negative rational numbers. Moreover, the map

$$
\mathcal{D}^{n, n-p} \ni \varphi \mapsto M_{f, \mathcal{I}}(\varphi, 0)
$$

defines the action of $a(0, p)$-current $T_{f, \mathcal{I}}$ on $V$ such that $\bar{h} T_{f, \mathcal{I}}=0$ for any $h \in \mathcal{O}(V)$ which vanishes on the common zero set

$$
\mathcal{Z}(f):=\left\{\zeta \in V ; f_{1}=\cdots=f_{m}=0\right\} .
$$

The current $T_{f, \mathcal{I}}$ is hence supported by $\mathcal{Z}(f)$, and moreover, one has

$$
T_{f, \mathcal{I}}=0
$$

when $p<\operatorname{codim} \mathcal{Z}(f)$.

Proof: Clearly one can reduce the problem to the case where the support of the test form is an arbitrarily small neighborhood of a point $z_{0}$ in $\mathcal{Z}(f)$, and for the sake of simplicity we will reduce ourselves, via a change of variables, to the case $z_{0}=0$. We will therefore assume that $\operatorname{Supp} \varphi \subset$ $W$, where $W$ is a neighborhood of the origin such that there exists a desingularisation $(\mathcal{X}, \Pi), \mathcal{X}$ being a $n$-dimensional complex manifold and $\Pi$ a proper holomorphic map $\mathcal{X} \rightarrow W$, such that

(i) the hypersurface $\Pi^{*}\left(\left\{f_{1} \cdots f_{m}=0\right\}\right)$ has normal crossings in $\mathcal{X}$;

(ii) the map $\pi$ is a biholomorphic map between $\mathcal{X} \backslash \Pi^{*}\left(\left\{f_{1} \cdots f_{m}=0\right\}\right)$ and $W \backslash\left\{f_{1} \cdots f_{m}=0\right\}$.

The existence of such a pair $(\mathcal{X}, \Pi)$ follows from Hironaka's theorem [20]. For $\operatorname{Re} \lambda$ sufficiently large, one can write $M_{f, \mathcal{I}}(\varphi, \lambda)$ as a finite sum of terms

$$
\int_{\omega} \lambda\left\|\Pi^{*} f\right\|^{2 \lambda} \Pi^{*} \Theta_{f, \mathcal{I}} \wedge \rho \Pi^{*} \varphi
$$


where

$$
\Theta_{f, \mathcal{I}}:=c_{p} \frac{\bar{\partial}\|f\|^{2} \wedge\left(\sum_{k=1}^{p}(-1)^{k-1} \overline{f_{i_{k}}} \bigwedge_{l \neq k} \overline{d f_{i_{l}}}\right)}{\|f\|^{2(p+1)}},
$$

$\omega$ is a local chart on $\mathcal{X}$, coming from a finite covering of the compact subset $\pi^{*}(\operatorname{Supp} \varphi)$, and $\rho$ is the function from the partition of unity (subordinate to the covering) which corresponds to the local chart $\omega$. Thanks to the normal crossing condition (i), one can assume that in a system of local coordinates on $\omega$ centered at the origin,

$$
\Pi^{*} f_{j}(t)=u_{j}(t) \prod_{k=1}^{n} t_{k}^{\alpha_{j k}}=u_{j}(t) m_{j}(t), \quad j=1, \ldots, m,
$$

where the $u_{j}$ are invertible elements in $\mathcal{O}(\omega)$ and the $\alpha_{j k}$ are positive integers. If one of the vectors $\alpha_{j}:=\left(\alpha_{j 1}, \ldots, \alpha_{j n}\right), j=1, \ldots, m$, is zero, the corresponding function of $\lambda$ in (2.4) is entire as $\lambda \mapsto\left\|\Pi^{*} f\right\|^{2 \lambda}$ is. So that the interesting case occurs when all the $\alpha_{j}$ are nonzero.

In order to study such a term, we use an idea that has already been extensively developed in [4]. Let $\Delta$ be the closed convex hull (in $\mathbf{R}_{+}^{n}$ ) of

$$
\bigcup_{j=1}^{m}\left\{\alpha_{j}+\mathbf{R}_{+}^{n}\right\}
$$

and $\triangleq$ the corresponding equivalence relation between elements in $\mathbf{R}_{+}^{n}$ : $\xi \stackrel{\Delta}{\sim} \xi^{\prime}$ if and only if $\operatorname{Tr}_{\Delta}(\xi)=\operatorname{Tr}_{\Delta}\left(\xi^{\prime}\right)$, where

$$
\operatorname{Tr}_{\Delta}(\xi):=\left\{\delta \in \Delta,\langle\xi, \delta\rangle=\min _{x \in \Delta}\langle\xi, x\rangle\right\} .
$$

(The brackets here stand for the usual scalar product in the affine space $\mathbf{R}^{n}$.) The set of all closures of the equivalence classes for this relation is a fan $\Sigma(\Delta)$ (see $[\mathbf{1}]$ and $[\mathbf{1 7}])$. Such a fan can be refined $([\mathbf{2 2}])$ in order that all cones are simple ones, so that the corresponding toric variety $\widetilde{\mathcal{X}}$ is a $n$-dimensional complex manifold; local charts correspond to different copies of $\mathbf{C}^{n}$ which are glued together via invertible monoidal transformations from the $n$-dimensional torus $\mathbf{T}^{n}$ into itself. Since the union of the cones in this fan is $\mathbf{R}_{+}^{n}$, the projection map $\widetilde{\Pi}: \widetilde{\mathcal{X}} \rightarrow \mathbf{C}^{n}$ (which is monoidal when expressed in local coordinates in each chart) is a proper map. Moreover, $\widetilde{\Pi}$ is invertible from $\widetilde{\mathcal{X}} \backslash \widetilde{\Pi}^{*}\left\{t_{1} \cdots t_{n}=0\right\}$ to $\mathbf{C}^{n} \backslash\left\{t_{1} \cdots t_{n}=0\right\}$. In each chart $\varpi$ on $\widetilde{\mathcal{X}}$ (the coordinates being $\left.\tau_{1}, \ldots, \tau_{n}\right)$, one can write

$$
\widetilde{\Pi}^{*} \Pi^{*} f_{j}\left(\tau_{1}, \ldots, \tau_{n}\right)=\left(\widetilde{\Pi}^{*} u_{j}\left(\tau_{1}, \ldots, \tau_{n}\right)\right) \mu_{j}(\tau), \quad j=1, \ldots, m,
$$


where $\mu_{j}=\widetilde{\Pi}^{*} m_{j}$ is also a monomial. Moreover, since the toric variety $\widetilde{\mathcal{X}}$ is associated with the Newton polyedron $\Delta$ attached to $\alpha_{1}, \ldots, \alpha_{m}$, there exists an index $j_{\varpi} \in\{1, \ldots, m\}$ such that $\mu_{\varpi}$ divides all monomials $\mu_{j}$, $j=1, \ldots, m$ (see $[\mathbf{1}]$ ). This implies that

$$
\widetilde{\Pi}^{*} \Pi^{*}\|f\|^{2}(\tau)=\tilde{u}(\tau)\left|\mu_{j_{\varpi}}(\tau)\right|^{2}, \quad \tau \in \varpi,
$$

where $\tilde{u}$ is a non-vanishing positive real analytic function in $\varpi$. Since $\widetilde{\Pi}^{*}$ and $\Pi^{*}$ commute with $\partial$ and $\bar{\partial}$, one has

$$
\begin{aligned}
& \lambda \int_{\omega}\left\|\Pi^{*} f\right\|^{2 \lambda} \Pi^{*} \Theta_{f, \mathcal{I}} \wedge \rho \Pi^{*} \varphi \\
& =\lambda \sum_{\varpi} \int_{\varpi} \frac{\left|u \mu_{j_{\varpi}}\right|^{2 \lambda}}{\mu_{j_{\varpi}}^{p}}\left(\tilde{\theta}_{\varpi, 1}+\tilde{\theta}_{\varpi, 2} \wedge \frac{\overline{d \mu_{j_{\varpi}}}}{\overline{\mu_{j_{\varpi}}}}\right) \wedge \xi_{\rho, \varpi} \widetilde{\Pi}^{*}\left(\rho \Pi^{*} \varphi\right),
\end{aligned}
$$

where the $\left(\xi_{\rho, \varpi}\right)_{\varpi}$ correspond to a smooth partition of unity subordinate to $\widetilde{\Pi}^{*}(\operatorname{Supp} \rho)$ and $\tilde{\theta}_{\varpi, 1}$ and $\tilde{\theta}_{\varpi, 2}$ are smooth forms of bidegree $(0, p)$ and $(0, p-1)$ respectively.

For any smooth functions $\psi \in \mathcal{D}(\Omega)$ and $v \in \mathcal{C}^{\infty}(\Omega)$, where $\Omega \subset \mathbf{C}$, such that $v>0$ on Supp $\psi$, one can see immediately, just integrating by parts, that the maps defined for $\operatorname{Re} \lambda>p$ by

$$
\begin{aligned}
& \lambda \mapsto \lambda \int_{\Omega} v^{\lambda}|s|^{2 \lambda} \psi(s) \frac{d \bar{s}}{\bar{s}} \wedge \frac{d s}{s^{p}} \\
& \lambda \mapsto \lambda \int_{\Omega} v^{\lambda}|s|^{2 \lambda} \psi(s) \frac{d \bar{s} \wedge d s}{s^{p}}
\end{aligned}
$$

extend to meromorphic maps with poles in $\{r \in \mathbf{Q}, r<0\}$. The value at $\lambda=0$ corresponds to the action of a distribution (with support at the origin) on the test function $\psi$ in the first case; the value at $\lambda=0$ is 0 in the second case. Moreover, the distribution that appears in the first case is annihilated by $\bar{s}$.

It follows from the above remark that each term in the right hand side of (2.6) can be meromorphically continued as a function of $\lambda$ with poles in $\{r \in \mathbf{Q}, r<0\}$. The value at the origin of the meromorphic continuation of any function of the form (2.6) corresponds to the action of a $(0, p)$-current in $V$. Summing up all functions of $\lambda$ of the form (2.6), we find that the function $\lambda \mapsto M_{f, \mathcal{I}}(\varphi, \lambda)$ can be meromorphically continued to the whole plane, with strictly negative rational poles. The value at $\lambda=0$ corresponds to the action of a $(0, p)$-current $T_{f, \mathcal{I}}$. 
Suppose that $h \in \mathcal{O}(V), h=0$ on $V$. It follows from the Nullstellensatz that for any $\varphi \in \mathcal{D}^{n, n-p}(V)$, one has $h^{N(\varphi)} \in\left(f_{1}, \ldots, f_{m}\right)_{\text {loc }}$ for some integer $N(\varphi)$. For any $\Pi$ and $\widetilde{\Pi}$ involved in the resolutions of singularities used in the proof, and for any $\rho, \varpi, \xi_{\rho, \varpi}$ as before, we have the estimate

$$
\left|\widetilde{\Pi}^{*} \Pi^{*}(\bar{h})(\tau)\right|^{N(\varphi)} \leq C\left(\xi_{\rho, \varpi}\right) \widetilde{\Pi}^{*} \Pi^{*}\|f\|^{2}(\tau) \leq \widetilde{C}\left(\xi_{\rho, \varpi}\right)\left|\mu_{j_{\varpi}}(\tau)\right|, \quad \tau \in \varpi,
$$

which implies that any $\tau_{k}, k=1, \ldots, n$, which divides $\mu_{j_{\varpi}}$ also divides $\widetilde{\Pi}^{*} \Pi^{*} h$. This means that, for any $\varphi \in \mathcal{D}(V)$,

$$
\begin{aligned}
& {\left[\lambda \sum_{\varpi} \int_{\varpi} \frac{\left|\tilde{u} \mu_{j_{\varpi}}\right|^{2 \lambda}}{\mu_{j_{\varpi}}^{p}}\left(\tilde{\theta}_{\varpi, 1}+\tilde{\theta}_{\varpi, 2} \wedge \frac{\overline{d \mu_{j_{\varpi}}}}{\overline{\mu_{j_{\varpi}}}}\right) \wedge \xi_{\rho, \varpi} \widetilde{\Pi}^{*}\left(\rho \Pi^{*} \bar{h} \varphi\right)\right]_{\lambda=0} } \\
= & {\left.\left[\lambda \sum_{\varpi} \int_{\varpi} \frac{\left|\tilde{u} \mu_{j_{\varpi}}\right|^{2 \lambda}}{\mu_{j_{\varpi}}^{p}} \tilde{\theta}_{\varpi, 2} \wedge \frac{\left(\widetilde{\Pi}^{*} \Pi^{*} \bar{h}\right) \overline{d \mu_{j_{\varpi}}}}{\overline{\mu_{j_{\varpi}}}}\right) \wedge \xi_{\rho, \varpi} \widetilde{\Pi}^{*}\left(\rho \Pi^{*} \varphi\right)\right]_{\lambda=0}=0 }
\end{aligned}
$$

because the differential form

$$
\frac{\left(\widetilde{\Pi}^{*} \Pi^{*} \bar{h}\right) \overline{d \mu_{j_{\varpi}}}}{\overline{\mu_{j_{\varpi}}}}
$$

is nonsingular.

In order to prove the last assertion in the statement of Lemma 2.2, assume $p<\operatorname{codim}\left\{f_{1}=\cdots=f_{m}=0\right\}$ and take a test form $\varphi \in$ $\mathcal{D}^{n, n-p}(V)$. One can rewrite $\varphi$ as

$$
\varphi=\sum_{1 \leq i_{1}<\cdots<i_{n-p} \leq n} \varphi_{i_{1}, \ldots, i_{n-p}} d \zeta_{1} \wedge \cdots \wedge d \zeta_{n} \wedge \bigwedge_{l=1}^{n-p} \overline{d \zeta_{i_{l}}}
$$

Each differential form $\bigwedge_{l=1}^{n-p} \overline{d \zeta_{i_{l}}}$ is zero when restricted to the analytic variety $V \cap\left\{f_{1}=\cdots=f_{m}=0\right\}$. This implies that, given a local chart $\varpi$ on any toric manifold such as $\tilde{\mathcal{X}}$, the differential form $\widetilde{\Pi}^{*} \Pi^{*} \bigwedge_{l=1}^{n-p} \overline{d \zeta_{i_{l}}}$ (which has antiholomorphic functions as coefficients) vanishes on the analytic variety $\left\{\mu_{j_{\varpi}}(\tau)=0\right\}$, where $\mu_{j_{\varpi}}$ is the distinguished monomial corresponding to the local chart $\varpi$. Every conjugate coordinate $\bar{\tau}_{k}$, such that $\tau_{k}$ is involved in $\mu_{j_{\varpi}}$ then divides each coefficient of $\widetilde{\Pi}^{*} \Pi^{*} \bigwedge_{l=1}^{n-p} \overline{d \zeta_{i_{l}}}$, which does not contain $d \bar{\tau}_{k}$. This implies that for any local chart $\varpi$, the integrand in (2.5) does not contain antiholomorphic singularities (such singularities come from logarithmic derivatives and therefore are cancelled by the corresponding term $\widetilde{\Pi}^{*} \Pi^{*} \varphi$ ). The proof of our Lemma 2.2 is complete. 
Let us recall the definition of the integral closure of an ideal $\mathcal{A}$ in the ring ${ }_{n} \mathcal{O}_{z_{0}}$ of germs of holomorphic functions of $n$ variables at a point $z_{0} \in \mathbf{C}^{n}$. A germ $h$ at $z_{0}$ is in the integral closure of $\mathcal{A}$ if and only if it satisfies a relation of integral dependency

$$
h^{N}+\sum_{k=1}^{N} a_{k} h^{N-k}=0,
$$

where $a_{k} \in \mathcal{A}^{k}$ for each $k \in\{1, \ldots, N\}$. If $V$ is an open set in $\mathbf{C}^{n}$ and $A$ an ideal in $\mathcal{O}(V)$, an element $h \in \mathcal{O}(V)$ is locally in the integral closure of $A$ if and only if, at any point $z_{0} \in V$, the germ $h_{z_{0}}$ belongs to the integral closure (in ${ }_{n} \mathcal{O}_{z_{0}}$ ) of the ideal $\mathcal{A}_{z_{0}}$ generated by the germs at $z_{0}$ of all elements in $A$.

Lemma 2.3. Let $T_{f, \mathcal{I}}$ be the current occurring in the preceeding lemma. For any $h \in \mathcal{O}(V)$ which is locally in the integral closure of the ideal $\left(f_{1}, \ldots, f_{m}\right)^{p}$ we then have

$$
h T_{f, \mathcal{I}}=0 .
$$

Proof: Replacing $\varphi$ by $h \varphi$ and arguing as in the proof of Lemma 2.2, we decompose the function $\lambda \mapsto M_{f, \mathcal{I}}(h \varphi, \lambda)$ into a finite sum of expressions of the type (2.5) (modulo an entire function which vanishes at the origin). The only thing we have to show is that, for any $h$ which locally belongs to the integral closure of $\left(f_{1}, \ldots, f_{m}\right)^{p}$ in $\mathcal{O}(V)$, the value at $\lambda=0$ of the analytic continuation of

$$
\lambda \mapsto \int_{\varpi} \frac{\left|\tilde{u} \mu_{j_{\varpi}}\right|^{2 \lambda}}{\mu_{j_{\varpi}}^{p}}\left(\tilde{\theta}_{\varpi, 1}+\tilde{\theta}_{\varpi, 2} \wedge \frac{\overline{d \mu_{j_{\varpi}}}}{\overline{\mu_{j_{\varpi}}}}\right) \wedge \xi_{\rho, \varpi} \widetilde{\Pi}^{*}\left(\rho \Pi^{*} h \varphi\right)
$$

equals zero. (The notations are those from the proof of Lemma 2.2.) Since $h$ is locally in the integral closure of $\left(f_{1}, \ldots, f_{m}\right)^{p}$, it follows, from the existence of local relations of algebraic dependency (2.7) of $h$ over the ideal $\left(f_{1}, \ldots, f_{m}\right)^{p}$, that near any point $z_{0} \in V$, one has a local estimate

$$
|h(\zeta)| \leq C_{z_{0}}\left(\max _{1 \leq j \leq m}\left|f_{j}(\zeta)\right|\right)^{p} .
$$

Such local estimates imply that in any local chart $\varpi$ involved in (2.5), one has, on the support of $\xi_{\rho, \varpi}$

$$
\left|\widetilde{\Pi}^{*} \Pi^{*} h\right| \leq C_{\rho, \varpi} \widetilde{\Pi}^{*} \Pi^{*}\|f\|^{p} \leq \widetilde{C}_{\rho, \varpi}\left|\mu_{j_{\varpi}}\right|^{p} .
$$


This implies that the monomial $\mu_{j_{\varpi}}$ divides $\widetilde{\Pi}^{*} \Pi^{*} h$. We now make the following observation: for any domain $\Omega \subset \mathbf{C}$, and any smooth functions $\psi \in \mathcal{D}(\Omega)$ and $v \in \mathcal{C}^{\infty}(\Omega)$, such that $v>0$ on $\operatorname{Supp} \psi$, explicit integration by parts provides meromorphic continuations of the maps defined for $\operatorname{Re} \lambda>p$ by

$$
\begin{aligned}
\lambda & \mapsto \lambda \int_{\Omega} v^{\lambda}|s|^{2 \lambda} \psi(s) d \bar{s} \wedge d s \\
\lambda & \mapsto \lambda \int_{\Omega} v^{\lambda}|s|^{2 \lambda} \psi(s) \frac{d \bar{s} \wedge d s}{\bar{s}} .
\end{aligned}
$$

Their poles are again strictly negative rational numbers, and they both vanish for $\lambda=0$. This shows that the value at $\lambda=0$ of the analytic continuation of any of the functions

$$
\lambda \mapsto \int_{\varpi} \frac{\tilde{u}\left|\mu_{j_{\varpi}}\right|^{2 \lambda}}{\mu_{j_{\varpi}}^{p}}\left(\tilde{\theta}_{\varpi, 1}+\tilde{\theta}_{\varpi, 2} \wedge \frac{\overline{d \mu_{j_{\varpi}}}}{\overline{\mu_{j_{\varpi}}}}\right) \wedge \xi_{\rho, \varpi} \widetilde{\Pi}^{*}\left(\rho \Pi^{*} h \varphi\right)
$$

is also equal to zero. Since our original function of $\lambda$ (as in (2.2), but with $h \varphi$ instead of $\varphi$ ) is a combination of such expressions, its analytic continuation also vanishes at the origin. This proves our result.

The last lemma that we will need in order to conclude the proof of our Theorem 1.1 is concerned with rapid decrease in imaginary directions.

Lemma 2.4. Let $V$ be an open set in $\mathbf{C}^{n}$ and let $f_{1}, \ldots, f_{m}$ be elements in $\mathcal{O}(V)$. Let $\theta$ be any test form in $V$ of maximal bidegree $(n, n)$ and denote by $\lambda_{\theta, 0}>\lambda_{\theta, 1}>\ldots$ the sequence of all poles (necessarily in $\{r \in \mathbf{Q}, r<0\}$ ) of the meromorphic continuation $F_{\theta}$ of

$$
\lambda \mapsto \int_{V}\left(\left|f_{1}\right|^{2}+\cdots+\left|f_{m}\right|^{2}\right)^{\lambda} \theta .
$$

Then, for any natural number $k$ and any real numbers $\alpha, \beta$, such that $\lambda_{\theta, j+1}<\alpha<\beta<\lambda_{\theta, j}, j \in \mathbf{N}^{*}$ or $\lambda_{\theta, 0}<\alpha<\beta$, there is a constant $\gamma(k, \alpha, \beta)$ such that

$$
\sup _{\alpha \leq \operatorname{Re} \lambda \leq \beta}\left|(1+|\lambda|)^{k} F_{\theta}(\lambda)\right| \leq \gamma(k, \alpha, \beta) .
$$

(In other words, the function $F_{\theta}$ is rapidly decreasing at infinity in any closed vertical strip which is free of poles.) 
Proof: Our proof was inpired by an argument used in [2]. Let $G$ be the holomorphic function in $V \times V$ defined as

$$
G(z, w):=\sum_{j=1}^{m} f_{j}(z) \overline{f_{j}(\bar{w})} .
$$

Consider a point $z_{0}$ in $V$ where $f_{j}\left(z_{0}\right)=0, j=1, \ldots, m$. By Corollary 9.10 in Chapter 5 of [10], there exists a neighborhood $\mathcal{V}\left(z_{0}\right)$ of $\left(z_{0}, z_{0}\right)$ in $V \times V$ such that $\mathcal{V}\left(z_{0}\right) \cap\{d G=0\} \subset \mathcal{V}\left(z_{0}\right) \cap\{G=0\}$. As was proved in $[\mathbf{2 1}$, Section 6$]$, there is then an operator $P_{z_{0}}(\lambda, z, w, \partial / \partial z, \partial / \partial w)$ in $\mathcal{D}_{\left(z_{0}, z_{0}\right)}[\lambda]$, where $\mathcal{D}_{\left(z_{0}, z_{0}\right)}$ denotes the ring of holomorphic differential operators in $2 n$ complex variables with coefficients in the $\operatorname{ring}{ }_{2 n} \mathcal{O}_{\left(z_{0}, z_{0}\right)}$ of germs of holomorphic functions at the point $\left(z_{0}, w_{0}\right)$, such that

$$
P_{z_{0}}(\lambda, z, w, \partial / \partial z, \partial / \partial w)=\lambda^{M}-\sum_{l=1}^{M} \lambda^{M-l} P_{z_{0}, l}(z, w, \partial / \partial z, \partial / \partial w)
$$

and

$$
P_{z_{0}}(\lambda, z, w, \partial / \partial z, \partial / \partial w)\left[G^{\lambda}\right]=0 .
$$

If we now make the substitution $w=\bar{z}$, and use the fact that the operators $\partial / \partial z_{l}$ and $\partial / \partial \bar{z}_{l}$ commute with multiplication by $\bar{z}_{k}$ and $z_{k}$, respectively, we find that in a neighborhood $V\left(z_{0}\right)$ of $z_{0}$, there holds the identity (in the sense of distributions)

$$
P_{z_{0}}(\lambda, z, \bar{z}, \partial / \partial z, \partial / \partial \bar{z})\left[\left(\sum_{j=1}^{m}\left|f_{j}\right|^{2}\right)^{\lambda}\right]=0 .
$$

This functional equation (2.12), used in the form

$$
\left[\left(\sum_{j=1}^{m}\left|f_{j}\right|^{2}\right)^{\lambda}\right]=\sum_{l=1}^{M} \frac{P_{z_{0}, l}(z, \bar{z}, \partial / \partial z, \partial / \partial \bar{z})}{\lambda^{l}}\left[\left(\sum_{j=1}^{m}\left|f_{j}\right|^{2}\right)^{\lambda}\right]
$$

and then iterated (as in an argument quoted from [2]), provides the rapid decrease of $F_{\theta}$ on closed vertical strips in the $\lambda$-plane which are pole-free. Notice that the fact that the meromorphic continuation of $F_{\theta}$ exists (with poles organized as a decreasing sequence of strictly negative rational numbers) follows (as in our proof of Lemma 2.1) from Hironaka's theorem on resolution of singularities. The proof of Lemma 2.3 is thereby complete. 
Proof of Theorem 1.1: We have now collected all elements for the proof of our Theorem 1.1. Recall that the Mellin transform of the function $\eta \mapsto$ $J_{f, \mathcal{I}}(\varphi, \eta)$ defined as in $(2.1)$, is equal to the function $\lambda \mapsto M_{f, \mathcal{I}}(\varphi, \lambda)$ described in Lemma 2.1. The Fourier-Laplace inversion formula then tells us that, for $\gamma_{0}>0$ large enough, the identity

$$
J_{f, \mathcal{I}}(\varphi, \eta)=\frac{1}{2 \pi i} \int_{\gamma_{0}+i \mathbf{R}}\left(M_{f, \mathcal{I}}(\varphi, \lambda) \eta^{-\lambda} d \lambda\right) / \lambda
$$

holds for every positive $\eta$. We know from Lemma 2.2 that there is a positive number $\varepsilon_{0}$ such that the only pole of

$$
\lambda \mapsto \frac{J_{f, \mathcal{I}}(\mathcal{I} ; \varphi, \lambda) \eta^{-\lambda}}{\lambda}
$$

in the closed vertical strip $\Gamma:=\operatorname{Re} \lambda \in\left[-\varepsilon_{0}, \gamma_{0}\right]$ is the origin, and that the residue is $M_{f, \mathcal{I}}(\varphi, 0)$. It follows from Lemma 2.4 that the function (2.13) is rapidly decreasing at infinity on the strip $\Gamma$. We can apply the residue formula and get that $J_{f, \mathcal{I}}(\varphi, \eta)$ is equal to

$$
M_{f, \mathcal{I}}(\varphi, 0)+\frac{1}{2 \pi i} \int_{-\varepsilon_{0}+i \mathbf{R}} \frac{M_{f, \mathcal{I}}(\varphi, \lambda) \eta^{-\lambda}}{\lambda} d \lambda=M_{f, \mathcal{I}}(\varphi, 0)+O\left(\eta^{\varepsilon_{0}}\right) .
$$

We conclude that the limit (1.4) exists and equals $M_{f, \mathcal{I}}(\varphi, 0)$. We get the conclusions (i) and (iii) of Theorem 1.1 from Lemma 2.2, and conclusion (ii) from Lemma 2.3. Our main Theorem 1.1 is thus proved.

One can also realize the action of all the currents $T_{f, \mathcal{I}}$ in Theorem 1.1 as limits of solid volume integrals. More precisely, we have the following theorem:

Theorem 2.1. Let $f_{1}, \ldots, f_{m}$ be holomorphic functions in some open set $V \in \mathbf{C}^{n}$. For any ordered subset $\mathcal{I} \subset\{1, \ldots, m\}$ of cardinality $p \leq \min (m, n)$, let $T_{f, \mathcal{I}}$ be the current defined in (1.4). Then one has the representation

$$
T_{f, \mathcal{I}}(\varphi)=\lim _{\tau \rightarrow 0^{+}} c_{p} p \int_{V} \frac{\tau \bar{\partial}\|f\|^{2} \wedge\left[\sum_{k=1}^{p}(-1)^{k-1} \overline{f_{i_{k}}} \bigwedge_{l \neq k} \overline{d f_{i_{l}}}\right] \wedge \varphi}{\|f\|^{2}\left(\|f\|^{2}+\tau\right)^{p+1}}
$$

In particular, if $m \leq n$ and $\mathcal{I}=\{1, \ldots, m\}$, we have

$$
T_{f, \mathcal{I}}(\varphi)=\lim _{\tau \rightarrow 0^{+}} c_{m} m \int_{V} \frac{\tau \bigwedge_{k=1}^{m} \overline{d f_{k}} \wedge \varphi}{\left(\|f\|^{2}+\tau\right)^{m+1}} .
$$


Proof: Notice first that the integral in (2.14) is absolutely convergent, for the differential form inside the integral has bounded coefficients. Let us fix $\tau>0$. If $[\alpha, \beta]$ denotes any interval in $\mathbf{R}_{+}$which does not contain a critical value for the mapping $\operatorname{Supp} \varphi \ni \zeta \mapsto\|f(\zeta)\|^{2}$, it follows from Fubini's theorem that

$$
\begin{aligned}
p \tau & \int_{[\alpha, \beta]} \frac{\eta^{p-1} J_{f}(\mathcal{I} ; \varphi, \eta)}{(\eta+\tau)^{p+1}} d \eta \\
& =c_{p} p \tau \int_{[\alpha, \beta]}\left(\int_{\|f\|^{2}=\eta}\left[\sum_{k=1}^{p}(-1)^{k-1} \overline{f_{i_{k}}} \bigwedge_{l \neq k} \overline{d f_{i_{l}}}\right] \wedge \varphi\right) \frac{d \eta}{\eta(\eta+\tau)^{p+1}} \\
& =c_{p} p \tau \int_{V \cap\left\{\alpha \leq\|f\|^{2} \leq \beta\right\}} \frac{\bar{\partial}\|f\|^{2} \wedge\left[\sum_{k=1}^{p}(-1)^{k-1} \overline{f_{i_{k}}} \bigwedge_{l \neq k} \overline{d f_{i_{l}}}\right] \wedge \varphi}{\|f\|^{2}\left(\|f\|^{2}+\tau\right)^{p+1}}
\end{aligned}
$$

Since the critical values for $\|f\|^{2}$ which are attained on $\operatorname{Supp} \varphi$ form a negligible closed subset of $\mathbf{R}_{+}$, we get from Lebesgue's theorem and from the continuity at $\eta=0$ of $\eta \mapsto J_{f, \mathcal{I}}(\varphi, \eta)$ that for any $\tau>0$

$$
\begin{aligned}
& p \tau \int_{0}^{\infty} \frac{\eta^{p-1} J_{f, \mathcal{I}}(\varphi, \eta)}{(\eta+\tau)^{p+1}} d \eta \\
& \quad=c_{p} p \tau \int_{V} \frac{\bar{\partial}\|f\|^{2} \wedge\left[\sum_{k=1}^{p}(-1)^{k-1} \overline{f_{i_{k}}} \bigwedge_{l \neq k} \overline{d f_{i_{l}}}\right] \wedge \varphi}{\|f\|^{2}\left(\|f\|^{2}+\tau\right)^{p+1}}
\end{aligned}
$$

We just note that for $\tau>0$,

$$
\begin{aligned}
\tau \int_{0}^{\infty} & \frac{\eta^{p-1} d \eta}{(\eta+\tau)^{p+1}} \\
& =\int_{\tau}^{\infty} \eta^{-p-1}\left(\tau^{p-1}+\sum_{k=1}^{p-1}(-1)^{k}\left(\begin{array}{c}
p-1 \\
k
\end{array}\right) \eta^{k} \tau^{p-k}\right) d \eta \\
& =1 / p+\rho(\tau)
\end{aligned}
$$

where $\lim _{\tau \rightarrow 0} \rho(\tau)=0$. Using the short-hand notation $J(\eta):=J_{f, \mathcal{I}}(\varphi, \eta)$ 
we can now rewrite the right hand side of (2.17) as

$$
\begin{aligned}
& p \tau \int_{0}^{\infty} \frac{\eta^{p-1} J(\eta) d \eta}{(\eta+\tau)^{p+1}} \\
&=J(0)+p \tau \int_{0}^{A} \frac{\eta^{p-1}(J(\eta)-J(0)) d \eta}{(\eta+\tau)^{p+1}} \\
&-p J(0)\left(\rho(\tau)+\tau \int_{A}^{\infty} \frac{\eta^{p-1} d t}{(\eta+\tau)^{p+1}}\right) \\
&=J(0)+p \tau \int_{0}^{\varepsilon} \frac{\eta^{p-1}(J(\eta)-J(0)) d \eta}{(\eta+\tau)^{p+1}} \\
&+p \tau \int_{\varepsilon}^{A} \frac{\eta^{p-1}(J(\eta)-J(0)) d t}{(\eta+\tau)^{p+1}}+\tilde{\rho}_{\varepsilon, A}(\tau) \\
&=J(0)+p \tau \int_{0}^{\varepsilon} \frac{\eta^{p-1}(J(\eta)-J(0)) d \eta}{(\eta+\tau)^{p+1}}+\check{\rho}_{\varepsilon, A}(\tau)
\end{aligned}
$$

for $\varepsilon<A$ and $\varepsilon$ arbitrary small, with $\lim _{\tau \rightarrow 0^{+}} \check{\rho}_{\varepsilon, A}(\tau)=0$. Since we can choose $\varepsilon$ arbitrarily small, we have

$$
\lim _{\tau \rightarrow 0^{+}} p \tau \int_{0}^{\infty} \frac{\eta^{p-1} J(\eta)}{(\eta+\tau)^{p+1}} d \eta=J(0)=T_{f, \mathcal{I}}(\varphi) .
$$

The conclusion of our theorem follows from (2.16) and (2.19). The final assertion in Theorem 2.1 follows from the fact that, if $m \leq n$ and $\mathcal{I}=\{1, \ldots, m\}$, then

$$
\bar{\partial}\|f\|^{2} \wedge\left[\sum_{k=1}^{m}(-1)^{k-1} \overline{f_{k}} \bigwedge_{l \neq k} \overline{d f_{l}}\right]=\|f\|^{2} \bigwedge_{k=1}^{m} \overline{d f_{k}} .
$$

This concludes the proof of Theorem 2.1.

When $m \leq n$, it is well known (see [5] or [24]) that for any test form $\varphi \in \mathcal{D}^{n, n-m}(V)$, the function

$$
\left(\lambda_{1}, \ldots, \lambda_{m}\right):=\lambda \mapsto \Gamma_{f}(\lambda, \varphi):=\frac{c_{m}}{(m-1) !} \int_{V} \prod_{k=1}^{m}\left|f_{k}\right|^{2\left(\lambda_{k}-1\right)} \bigwedge_{k=1}^{m} \overline{d f_{k}} \wedge \varphi
$$

can be continued from the cone $\operatorname{Re} \lambda_{j}>1,1 \leq j \leq n$, to a meromorphic function in the entire space $\mathbf{C}^{m}$, with polar set Sing $\Gamma_{f}$ included in a union of hyperplanes $\beta_{0}+\beta_{1} \lambda_{1}+\cdots+\beta_{m} \lambda_{m}=0$, where $\beta_{0} \in \mathbf{N}$, $\left(\beta_{1}, \ldots, \beta_{m}\right) \in \mathbf{N}^{m} \backslash\{0\}$. This is obtained immediately using Hironaka's theorem $[\mathbf{2 0}]$. It seems interesting to relate this meromorphic continuation $\lambda \mapsto \Gamma_{f}(\lambda, \varphi)$ to the computation of the residue currents we introduced in Theorem 1.1. We have the following result in this direction. 
Theorem 2.2. Let $m \leq n$ and take $\mathcal{I}=\{1, \ldots, m\}$. If $T_{f, \mathcal{I}}$ is the current defined in Theorem 1.1, then for any test form $\varphi \in \mathcal{D}^{n, n-m}(V)$, and for any $\left.\gamma=\left(\gamma_{1}, \ldots, \gamma_{m}\right) \in\right] 0,1\left[^{m}\right.$, one has

$$
\begin{aligned}
& T_{f, \mathcal{I}}(\varphi) \\
= & \lim _{\tau \rightarrow 0^{+}} \frac{1}{(2 \pi i)^{m}} \int_{\gamma+i \mathbf{R}^{m}} \tau^{-|s|} \Gamma(|s|+1) \prod_{k=1}^{m} \Gamma\left(1-s_{k}\right) \Gamma_{f}(s ; \varphi) d s,
\end{aligned}
$$

where $|s|:=s_{1}+\cdots+s_{m}$. Moreover, if $C$ is any connected component in $\mathbf{R}^{m}$ of the complement of $\operatorname{proj}_{\mathbf{R}^{m}}\left(\operatorname{Sing}\left(\Gamma_{f}\right)\right)$, then for any fixed $\tau>0$, and for any $\gamma \in C$, the integral

$$
S(C, \tau):=\int_{\gamma+i \mathbf{R}^{m}} \tau^{-|s|} \Gamma(|s|+1) \prod_{k=1}^{m} \Gamma\left(1-s_{k}\right) \Gamma_{f}(s ; \varphi) d s
$$

is absolutely convergent.

Proof: This result was proved in [29] in the complete intersection case. In fact, this hypothesis is not necessary and the whole proof goes through as follows. Fix $\tau>0$. For any $\zeta$ in $V$ such that $f_{1} \cdots f_{m}(\zeta) \neq 0$, one has, if $\tilde{\gamma}_{1}, \ldots, \tilde{\gamma}_{m}$ are strictly positive numbers with sum strictly less than $m$,

$$
=\frac{1}{(2 \pi i)^{m}} \int_{\tilde{\gamma}+i \mathbf{R}^{m}} \Gamma(m+1-|s|) \prod_{k=1}^{m} \Gamma\left(s_{k}\right) \prod_{k=1}^{m}\left|f_{k}(\zeta)\right|^{-2 s_{k}} \tau^{|s|-m} d s .
$$

This is just a standard iteration of formula 6.422 (3), p. 657 in [18]. Notice that this idea has been extensively used in [6]. If we change $s_{k}$ into $1-s_{k}$ and let $\gamma_{k}=1-\tilde{\gamma}_{k}, k=1, \ldots, m$, formula (2.22) can be rewritten as

$$
\begin{aligned}
& \left(m ! \tau /\left(\|f(\zeta)\|^{2}+\tau\right)^{m+1}\right) \\
& =\frac{1}{(2 \pi i)^{m}} \int_{\gamma+i \mathbf{R}^{m}} \Gamma(|s|+1) \prod_{k=1}^{m} \Gamma\left(1-s_{k}\right) \prod_{k=1}^{m}\left|f_{k}(\zeta)\right|^{2\left(s_{k}-1\right)} \tau^{-|s|} d s .
\end{aligned}
$$

If we assume that the $\gamma_{j}$ are all very close to 1 , we can apply Lebesgue's and Fubini's theorems in order to get, for such $\tau$,

$$
\begin{array}{rl}
c_{m} & m \tau \int_{V} \frac{\bigwedge_{k=1}^{m} \overline{d f_{k}} \wedge \varphi}{\left(\|f(\zeta)\|^{2}+\tau\right)^{m+1}} \\
& =\frac{1}{(2 \pi i)^{m}} \int_{\gamma+i \mathbf{R}^{m}} \Gamma(|s|+1) \prod_{k=1}^{m} \Gamma\left(1-s_{k}\right) \Gamma_{f}(s, \varphi) \tau^{-|s|} d s .
\end{array}
$$


Using Bernstein-Sato functional identities (see [28]) or resolution of singularities (which leads us to the normal crossing case) together with integration by parts, one can see that the function

$$
s \in \mathbf{C}^{m} \mapsto \Gamma_{f}(s, \varphi)
$$

can be estimated by

$$
\left|\Gamma_{f}(s, \varphi)\right| \leq C(\operatorname{Re} s)(1+\|\operatorname{Im} s\|)^{N(\operatorname{Re} s)},
$$

in any vertical strip $\operatorname{Re} s \in K, K \subset \subset \mathbf{R}^{m}$, which does not intersect the polar set of this function (in particular when $K \subset] 0, \infty{ }^{m}$ ), the constants $C(\operatorname{Re} s)$ and $N(\operatorname{Re} s)$ being uniform in $\operatorname{Re} s$ in this strip. Similar estimates hold for the function

$$
s \mapsto \Gamma(|s|+1) \Gamma_{f}(s, \varphi) .
$$

Therefore, because of the rapid decrease of the Gamma function on vertical lines, we get the uniform rapid decrease at infinity for the function

$$
s \mapsto \Gamma(|s|+1) \prod_{k=1}^{m} \Gamma\left(1-s_{k}\right) \Gamma_{f}(s, \varphi)
$$

in any vertical strip $\operatorname{Re} s \in K, K \subset \subset \mathbf{R}^{n}$ which is pole-free (in particular when $\left.K \subset] 0, \infty{ }^{m}\right)$. Thus, one can apply Cauchy's formula and replace $\left(\gamma_{1}, \ldots, \gamma_{m}\right)$ in $(2.23)$ by any element in $] 0,1{ }^{m}$. The first assertion of Theorem 2.2 follows from these computations, together with Theorem 2.1. The second assertion in the theorem is a consequence (in view of Cauchy's theorem) of the uniform rapid decrease at infinity in vertical strips (which are pole-free) for the function

$$
s \mapsto \Gamma(|s|+1) \prod_{k=1}^{m} \Gamma\left(1-s_{k}\right) \Gamma_{f}(s, \varphi) .
$$

Remark 2.1. Using the second part of this statement, it would be interesting to analyze how $S(C, \tau)$ changes when one moves from the original cell $] 0,1^{m}$ into the contiguous ones. The difference between $S\left(C_{1}, \tau\right)$ and $S\left(C_{2}, \tau\right)$ should appear (at least formally) as a (finite or infinite) sum of iterated residues for the function

$$
s \mapsto \tau^{-|s|} \Gamma(|s|+1) \prod_{k=1}^{m} \Gamma\left(1-s_{k}\right) \Gamma_{f}(s ; \varphi)
$$


relatively to collections of $m$ independent affine polar divisors. We will elaborate this idea somewhat in our computations in Section 3. Our assumption is motivated by the fact that for any $\tau>0$, for $\gamma>0$ very close to 0

$$
\begin{aligned}
& c_{m} m \tau \int_{V} \frac{\bigwedge_{k=1}^{m} \overline{d f_{k}} \wedge \varphi}{\left(\|f(\zeta)\|^{2}+\tau\right)^{m+1}} \\
& \quad=\frac{1}{2 \pi i} \int_{-\gamma+i \mathbf{R}} \Gamma(s) \Gamma(m+1-s) F(s, \varphi) \tau^{-m-s} d s
\end{aligned}
$$

where

$$
F(s, \varphi):=\frac{c_{m}}{(m-1) !} \int_{V}\|f\|^{2 s} \bigwedge_{k=1}^{m} \overline{d f_{k}} \wedge \varphi
$$

(this is proved as formula (2.23), just using formula $6.422(3)$, p. 657 in [18] this time without iterating it). Thanks to Lemma 2.4, we have the rapid decrease of $F$ (as a function of $s$ ) on vertical strips which are pole free. Using Cauchy's formula and moving the integration path in (2.24) to the left, we deduce from the fact that the poles of $F$ are in $]-\infty,-m[$ the existence of an asymptotic development for the function

$$
\tau \mapsto c_{m} m \tau \int_{V} \frac{\bigwedge_{k=1}^{m} \overline{d f_{k}} \wedge \varphi}{\left(\|f(\zeta)\|^{2}+\tau\right)^{m+1}}
$$

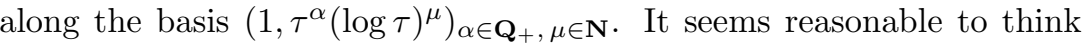
that the coefficients in this asymptotic development should be expressible as (infinite or finite) sums of residues corresponding to the meromorphic differential form

$$
\tau^{-|s|} \Gamma(|s|+1) \prod_{k=1}^{m} \Gamma\left(1-s_{k}\right) \Gamma_{f}(s ; \varphi) d s_{1} \wedge \cdots \wedge d s_{m} .
$$

This is precisely the point we will emphazise in the examples detailed in the next section.

\section{Some computations}

In this section, we will compute the action of some of the currents $T_{f, \mathcal{I}}$. Our approach is to deal only with the normal crossing case (even, in order to make things more simple, assume that the $f_{j}$ are all momomials) and profit (as we already did when we stated Theorem 2.2) from some combinatorial basic identities which correspond to multivariate analogs for the integral representation of the beta function as an inverse Mellin transform. 


\subsection{A simple example when $m=2$ and $f_{1}$ divides $f_{2}$.}

Assume that $n>1$ and $f_{1}$ and $f_{2}$ are defined in a neighborhood $V$ of the origin in $\mathbf{C}^{n}$. Let $f_{2}=f_{1} h$ and $\varphi \in \mathcal{D}^{n, n-2}(V)$. Then

$$
\begin{aligned}
M_{f,\{1,2\}}(\varphi, \lambda) & =\frac{\lambda}{4 \pi^{2}} \int_{V}\left|f_{1}\right|^{2(\lambda-2)}\left(1+|h|^{2}\right)^{\lambda-2} \overline{f_{1} d f_{1}} \wedge \overline{d h} \wedge \varphi \\
& =\frac{\lambda}{8 \pi^{2}} \int_{V}\left|f_{1}^{2}\right|^{(\lambda-2)}\left(1+|h|^{2}\right)^{\lambda-2} \overline{d f_{1}^{2}} \wedge \overline{d h} \wedge \varphi \\
& =M_{f_{1}^{2},\{1\}}\left(\left(1+|h|^{2}\right)^{\lambda-2} \overline{d h} \wedge \varphi, \frac{\lambda}{2}\right) .
\end{aligned}
$$

We conclude in this case that

$$
T_{f,\{1,2\}}(\varphi)=\left\langle\bar{\partial} \frac{1}{f_{1}^{2}} \wedge \frac{\overline{d h}}{\left(1+|h|^{2}\right)^{2}}, \varphi\right\rangle .
$$

This corresponds to the action of a current whose support is the zero set of the ideal $\left(f_{1}, f_{2}\right)$. Note that, in this case, the essential intersection (in the sense of [14]) of the divisors $\left\{f_{1}=0\right\}$ and $\left\{f_{2}=0\right\}$ (in this order) is empty, so that the Coleff-Herrera current associated to the sequence $\left(f_{1}, f_{2}\right)$ in this order would be zero. On the other hand, the Coleff-Herrera current associated to the sequence $\left(f_{2}, f_{1}\right)$ is the residual current $\bar{\partial} \frac{1}{\tilde{h}} \wedge \bar{\partial} \frac{1}{f_{1}}$, where $\tilde{h}$ is the product of irreducible factors in $h$ which are coprime with $f_{1}$. In any case, the Coleff-Herrera current for this example is either 0 , either a residual current supported by the origin and therefore differs from our current $T_{f,\{1,2\}}$.

3.2. The normal crossing case $m=n \geq 2$, and relations with Mellin-Barnes integrals.

In the space $\mathbf{C}^{n}$ we consider a system of monomials

$$
f=\left(f_{1}, \ldots, f_{n}\right)=\left(\zeta^{\alpha_{1}}, \ldots, \zeta^{\alpha_{n}}\right),
$$

and the Bochner-Martinelli type current (1.3) corresponding to this system. According to Theorem 2.2, this current may be represented as the limit

$$
\left.T_{f}(\varphi)=\lim _{\tau \rightarrow 0+} \frac{1}{(2 \pi i)^{n}} \int_{\gamma+i \mathbf{R}^{n}} \omega_{\tau}(s), \quad \gamma \in\right] 0,1\left[^{n},\right.
$$

where the integrand is given by the $n$-form

$$
\omega_{\tau}(s)=\tau^{-|s|} \Gamma(|s|+1) \prod_{k=1}^{n} \Gamma\left(1-s_{k}\right) \Gamma_{f}(s ; \varphi) d s .
$$


In the monomial case under consideration the possible poles of the function $s \mapsto \Gamma_{f}(s ; \varphi)$ consist of the $n$ families of hyperplanes

$$
\left\langle\alpha^{j}, s\right\rangle=0,-1,-2, \ldots ; \quad j=1, \ldots, n,
$$

where $\alpha^{j}$ denotes the $j^{\prime}$ th column vector in the matrix, whose row vectors are $\alpha_{1}, \ldots, \alpha_{n}$. In other words, if $\alpha_{i}=\left(\alpha_{i 1}, \ldots, \alpha_{i n}\right)$, then $\alpha^{j}=\left(\alpha_{1 j}, \ldots, \alpha_{n j}\right)$.

In the real subspace $\mathbf{R}^{n}$ with variables $x_{j}=\operatorname{Re} s_{j}, j=1, \ldots, n$, we now introduce the cone

$$
K=\left\{x \in \mathbf{R}^{n} ;\left\langle\alpha^{j}, x\right\rangle \geq 0, j=1, \ldots, n\right\}
$$

and we let $K_{0}$ denote the intersection of $K$ with the closed halfspace

$$
\Pi_{-}=\left\{x \in \mathbf{R}^{n} ; x_{1}+\cdots+x_{n} \leq 0\right\} .
$$

Let us write $q$ for the codimension of $K_{0}$. It is clear that if $q=0$, then $K_{0}$ will contain interior points of $\Pi_{-}$, whereas in the case $q \geq 1$ the intersection $K_{0}=K \cap \Pi_{-}$consists of a $(n-q)$-dimensional face of the cone $K$, contained in the hyperplane $x_{1}+\cdots+x_{n}=0$, i.e the boundary of $\Pi_{-}$. Up to a mere re-numbering of the faces, we may suppose that

$$
\begin{aligned}
K_{0} & =K_{0}^{q} \\
& =\left\{x \in \mathbf{R}^{n} ;\left\langle\alpha^{1}, x\right\rangle=\cdots=\left\langle\alpha^{q}, x\right\rangle=0,\left\langle\alpha^{q+1}, x\right\rangle \geq 0, \ldots,\left\langle\alpha^{n}, x\right\rangle \geq 0\right\} .
\end{aligned}
$$

We have the

Proposition 3.1. If $q=0$, then the current $T_{f}$, defined by (3.1), is equal to zero. In case $q \geq 1$, it admits the representation

$$
T_{f}=\bigwedge_{j=1}^{q} \bar{\partial}\left[\frac{1}{\zeta_{j}^{\left|\alpha^{j}\right|}}\right] \cdot \bigwedge_{k=q+1}^{n}\left(\frac{1}{\zeta_{k}^{\left|\alpha^{k}\right|}} \cdot \frac{d \overline{\zeta_{k}}}{\overline{\zeta_{k}}}\right) \cdot F\left(\left|\zeta_{q+1}\right|^{2}, \ldots,\left|\zeta_{n}\right|^{2}\right),
$$

where $\left|\alpha^{j}\right|$ denotes the sum of the components of the vector $\alpha^{j}$, and $F$ is a certain hypergeometric function (whose representation as a MellinBarnes integral is given in formula (3.8) below). In particular, if $q=n$, then

$$
T_{f}=\bar{\partial}\left[1 / \zeta_{1}^{\left|\alpha^{1}\right|}\right] \wedge \cdots \wedge \bar{\partial}\left[1 / \zeta_{n}^{\left|\alpha^{n}\right|}\right] .
$$

Remark 3.1. The complex codimension of the support of $T_{f}$ is equal to $q$, which is the real codimension of the cone $K_{0}$. 
Proof of the proposition: First of all we observe that by (the second statement of) Theorem 2.2 we may enlarge the cube $] 0,1\left[^{n}\right.$, consisting of admissible values for $\gamma$ in the integral (3.1), to the convex polyhedron $M$ obtained by intersecting the interior of the cone $K$ with the open cone $\left\{x \in \mathbf{R}^{n} ; x_{1}<1, \ldots, x_{n}<1\right\}$.

- If $q=0$, i.e $\operatorname{dim}_{\mathbf{R}^{n}} K_{0}=n$, then we can choose the point $\gamma$ in $M$ so that $|\gamma|<0$. Therefore, in view of the factor $\tau^{-|s|}$, the restriction of the form (3.2) to $\gamma+i \mathbf{R}^{n}$ tends to zero as $\tau \rightarrow 0^{+}$. It then follows that the limit (3.1) is equal to zero, and hence $T_{f}=0$.

- Assume now that $q \geq 1$. Letting $M^{q}$ denote the relative interior of the intersection $K_{0} \cap \bar{M}$, we have the following formula which decreases the number of integrations in (3.1).

Lemma 3.1. The limit of the $n$-fold integral (3.1) may be written as the $(n-q)$-fold integral

$$
T_{f}(\varphi)=\frac{1}{(2 \pi i)^{n-q}} \int_{\gamma^{q}+i \operatorname{Im} L^{q}} \prod_{j=1}^{n} \Gamma\left(1-s_{j}\right) \cdot \operatorname{Res}_{L^{q}}\left[\Gamma_{f}(s ; \varphi) d s\right]
$$

where $\gamma^{q} \in M^{q}$, and $\operatorname{Res}_{L^{q}}$ is the $q$-fold Poincaré-Leray residue class of the meromorphic form $\Gamma_{f} d s$, taken with respect to the intersection $L^{q}=$ $L_{1} \cap \cdots \cap L_{q}$ of the hyperplanes

$$
L_{j}=\left\{s \in \mathbf{C}^{n} ;\left\langle\alpha_{j}, s\right\rangle=0\right\}, \quad j=1, \ldots, q .
$$

To prove the lemma we establish first the following asymptotic (as $\tau \rightarrow 0^{+}$) formula:

$$
\frac{1}{(2 \pi i)^{n}} \int_{\gamma+i \mathbf{R}^{n}} \omega_{\tau}(s)=\frac{1}{(2 \pi i)^{n-1}} \int_{\gamma^{1}+i \operatorname{Im} L^{1}} \operatorname{Res}_{L^{1}} \omega_{\tau}(s)+o(\tau) .
$$

Here $\gamma^{1}$ is a point in the $(n-1)$-dimensional polyhedron $M_{1}$, which is the relative interior of the intersection $K_{0}^{1} \cap \bar{M} \subset L^{1}$. (Notice that $L^{1}=L_{1}$.) To this end we consider the ray

$$
\ell=\gamma+\left\{\left\langle\alpha^{1}, x\right\rangle \leq 0,\left\langle\alpha^{2}, x\right\rangle=\cdots=\left\langle\alpha^{n}, x\right\rangle=0\right\},
$$


emanating from the point $\gamma$ and parallel to one of the edges of the cone $K$. Now, what matters to us is the fact that this ray intersects the face $\left\langle\alpha^{1}, x\right\rangle=0$ no later than the hyperplane $|x|=0$ (the intersections occur simultaneously when $q=1$ ), and that among the polar hyperplanes of the form $s \mapsto \omega_{\tau}(s)$, the ray $\ell$ intersects only $L_{1}$. Letting $\gamma^{1}$ denote the point of intersection between $\ell$ and $\left\{x \in R^{n} ;\left\langle\alpha^{1}, x\right\rangle=0\right\}=\operatorname{Re} L_{1}$, we thus see that, for any two points $\gamma$ and $\kappa$ of $\ell$ lying on different sides of $\gamma^{1}$, the Cauchy formula yields

$$
\int_{\gamma+i \mathbf{R}^{n}} \omega_{\tau}(s)=\int_{\kappa+i \mathbf{R}^{n}} \omega_{\tau}(s)+2 \pi i \int_{\gamma^{1}+i \operatorname{Im} L^{1}} \operatorname{Res}_{L^{1}} \omega_{\tau}(s) .
$$

Choosing $\kappa$ lying inside $\Pi_{-}$, i.e with the property $|\kappa|<0$, we find, in view of the presence of the factor $\tau^{-|s|}$ in the form $\omega_{\tau}(s)$, that the integral over $\kappa+i \mathbf{R}^{n}$ in (3.6) tends to zero (is $o(\tau)$ ) as $\tau \rightarrow 0^{+}$. In this way we obtain (3.4).

In order to prove formula (3.3) we observe, that the integral in the right hand side of (3.4) has the same structure, but in the $(n-1)$-dimensional space $L_{1}$, so repeating $q-1$ times the residue theorem we arrive at the identity

$$
\lim _{\tau \rightarrow 0^{+}} \int_{\gamma+i \mathbf{R}^{n}} \omega_{\tau}(s)=(2 \pi i)^{q} \lim _{\tau \rightarrow 0^{+}} \int_{\gamma^{q}+i \operatorname{Im} L^{q}} \operatorname{Res}_{L^{q}} \omega_{\tau}(s)+o(\tau) .
$$

Since we assumed the face $K_{0}$ is contained in $\{|x|=0\}$, we have $L^{q} \subset$ $\{s ;|s|=0\}$, which means that the restriction of $s \mapsto \tau^{-|s|} \Gamma(1+|s|)$ to $L^{q}$ is identically equal to 1 . Recalling the expression (3.2) for the form $\omega_{\tau}$, we may thus conclude that

$$
\operatorname{Res}_{L^{q}} \omega_{\tau}(s)=\prod_{j=1}^{n} \Gamma\left(1-s_{j}\right)_{L^{q}} \cdot \operatorname{Res}_{L^{q}}\left[\Gamma_{f} d s\right]
$$

It follows from this that the right hand integral in (3.6) is actually independent of $\tau$. Hence there is no need to take a limit, and we have completed the proof of our lemma. 
Before applying formula (3.3) let us compute the iterated residue of the form $\Gamma_{f} d s$, where $\Gamma_{f}$ is given, for a monomial mapping $f$, by the integral

$$
\Gamma_{f}(s ; \varphi)=\frac{\delta}{(2 \pi i)^{n}} \int_{\mathbf{C}^{n}} \prod_{j=1}^{n}\left|\zeta_{j}\right|^{2\left(\left\langle\alpha^{j}, s\right\rangle-1\right)} \cdot \frac{\Phi(\zeta) \bigwedge_{j=1}^{n} d \bar{\zeta}_{j} \wedge d \zeta_{j}}{\prod_{j=1}^{n} \zeta_{j}^{\left|\alpha^{j}\right|-1}}
$$

where $\delta$ is the determinant of the matrix $\left(\alpha_{j k}\right)$, and the function $\Phi$ is related to the test form $\varphi$ by $\varphi=\Phi(\zeta) d \zeta$. In the new coordinates $\lambda=A s$, i.e $\lambda_{j}=\left\langle\alpha^{j}, s\right\rangle, j=1, \ldots, n$, we may represent $\Gamma_{f} d s$ as

$$
\Gamma_{f} d s=\frac{d \lambda_{1}}{\lambda_{1}} \wedge \cdots \wedge \frac{d \lambda_{q}}{\lambda_{q}} \wedge\left(R(\lambda) d \lambda^{\prime \prime}\right)+\vartheta(\lambda)
$$

where $\lambda=\left(\lambda^{\prime}, \lambda^{\prime \prime}\right)$, with $\lambda^{\prime}=\left(\lambda_{1}, \ldots, \lambda_{q}\right), \lambda^{\prime \prime}=\left(\lambda_{q+1}, \ldots, \lambda_{n}\right)$, and $\vartheta$ is a meromorphic $n$-form with poles along fewer (than $q$ ) of the hyperplanes $\lambda_{j}=0, j=1, \ldots, q$. Moreover, the form $\lambda^{\prime \prime} \mapsto R\left(0^{\prime}, \lambda^{\prime \prime}\right) d \lambda^{\prime \prime}$ is the desired iterated residue. This representation of $\Gamma_{f} d s$ is achieved by performing in (3.8) the integrations with respect to $\zeta^{\prime}=\left(\zeta_{1}, \ldots, \zeta_{q}\right)$, which can be accomplished through ordinary principal value integration (see $[\mathbf{2 5}]$ ), by means of polar coordinates and a Taylor expansion of $\Phi$.

An easy computation now leads to the following expresion:

$$
\begin{aligned}
& \Gamma_{f}\left(A^{-1}(\lambda) ; \varphi\right) \\
= & \frac{\delta}{(2 \pi i)^{n-q}} \cdot \frac{1}{\lambda_{1} \cdots \lambda_{q}}\left\{\int_{\mathbf{C}^{n}} \Phi_{\alpha}\left(\zeta^{\prime \prime}\right) \cdot \prod_{j=q+1}^{n} \frac{\left|\zeta_{j}\right|^{2\left(\lambda_{j}-1\right)}}{\zeta_{j}^{\left|\alpha^{j}\right|-1}} \bigwedge_{j=q+1}^{n} d \bar{\zeta}_{j} \wedge d \zeta_{j}+\tilde{\vartheta}(\lambda)\right\},
\end{aligned}
$$

where $\zeta=\left(\zeta^{\prime}, \zeta^{\prime \prime}\right), \tilde{\vartheta}$ is a holomorphic function in a neighborhood of the origin, belonging to the ideal $\left\langle\lambda_{1}, \ldots, \lambda_{q}\right\rangle$, and

$$
\Phi_{\alpha}\left(\zeta^{\prime \prime}\right)=\frac{1}{\left(\left|\alpha^{1}\right|-1\right) ! \cdots\left(\left|\alpha^{q}\right|-1\right) !} \frac{\partial^{\left|\alpha^{1}\right|+\cdots+\left|\alpha^{q}\right|-q}}{\partial \zeta_{1}^{\left|\alpha^{1}\right|-1} \cdots \partial \zeta_{q}^{\left|\alpha^{q}\right|-1}} \Phi\left(0^{\prime}, \zeta^{\prime \prime}\right) .
$$

Thus we get

$$
\begin{aligned}
\operatorname{Res}_{L^{q}}\left[\Gamma_{f} d s\right] & =R\left(0^{\prime}, \lambda^{\prime \prime}\right) d \lambda^{\prime \prime} \\
& =\frac{1}{(2 \pi i)^{n-q}} \int_{\mathbf{C}^{n-q}} \Phi_{\alpha}\left(\zeta^{\prime \prime}\right) \prod_{j=q+1}^{n} \frac{\left|\zeta_{j}\right|^{2 \lambda_{j}}}{\zeta_{j}^{\left|\alpha^{j}\right|}} \bigwedge_{j=q+1}^{n} \frac{d \bar{\zeta}_{j}}{\bar{\zeta}_{j}} \wedge d \zeta_{j} .
\end{aligned}
$$


Now, applying (3.1), (3.3) together with Fubini's theorem, we find that the action of the current $T_{f}$ may be expressed as

$T_{f}(\varphi)=\frac{1}{(2 \pi i)^{n-q}} \int_{\mathbf{C}^{n-q}} \frac{\Phi_{\alpha}\left(\zeta^{\prime \prime}\right)}{\prod_{j=q+1}^{n} \zeta_{j}^{\left|\alpha^{j}\right|}} \cdot F\left(\left|\zeta_{q+1}\right|^{2}, \ldots,\left|\zeta_{n}\right|^{2}\right) \bigwedge_{j=q+1}^{n} \frac{d \bar{\zeta}_{j}}{\bar{\zeta}_{j}} \wedge d \zeta_{j}$,

where $F$ is a function of the hypergeometric type, representable as the Mellin-Barnes integral

$$
\begin{aligned}
& F\left(\left|\zeta_{q+1}\right|^{2}, \ldots,\left|\zeta_{n}\right|^{2}\right) \\
& =\frac{1}{(2 \pi i)^{n-q}} \int_{i \mathbf{R}^{n-q}} \prod_{j=1}^{n} \Gamma\left(1-\ell_{j}\left(\lambda^{\prime \prime}\right)\right)\left|\zeta_{q+1}\right|^{2 \lambda_{q+1}} \cdots\left|\zeta_{n}\right|^{2 \lambda_{n}} d \lambda^{\prime \prime}
\end{aligned}
$$

where $\ell_{j}\left(\lambda^{\prime \prime}\right)$ is the $j^{\prime}$ th component of the vector $\ell\left(\lambda^{\prime \prime}\right)=A^{-1}\left(0^{\prime}, \lambda^{\prime \prime}\right)$. The proposition is thereby proved.

Remark 3.2. In case $q=1$ it is not hard to actually compute the integral (3.8) by using the methods of $[\mathbf{2 7}]$ and $[\mathbf{2 6}]$. The result of this computation then gives a rational function.

\section{The complete intersection case}

In this section, we consider $m \leq n$ holomorphic functions $f_{1}, \ldots, f_{m}$ defining a complete intersection in a domain $V \subset \mathbf{C}^{n}$. It follows from Theorem 1.1 (iii) that $\{1, \ldots, m\}$ is the only subset $\mathcal{I}$ that can give a nonzero current $T_{f, \mathcal{I}}$. We use the simpler notation $T_{f}$ for the corresponding current $T_{f,\{1, \ldots, m\}}$. We shall now prove that $T_{f}$ in fact coincides with the residue current in the sense of Coleff-Herrera ([14]).

Theorem 4.1. Let $f_{1}, \ldots, f_{m}$, be holomorphic functions defined in some open set $V \in \mathbf{C}^{n}$. Assume that $f_{1}, \ldots, f_{m}$ define a complete intersection in $V$ (in particular that $m \leq n)$. Then

$$
T_{f}=\bigwedge_{k=1}^{m} \bar{\partial} \frac{1}{f_{k}} .
$$

Proof: As mentioned in the introduction (see formula (1.2)), the actions of the two currents $T_{f}$ and $\bigwedge_{k=1}^{m} \bar{\partial} \frac{1}{f_{k}}$ on test forms which are $\bar{\partial}$-closed in a neighborhood of $\left\{f_{1}=\cdots=f_{m}=0\right\}$ coincide. The problem is to show this remains true for any test form. For this, we will need two preparatory lemmas. 
Lemma 4.1 ([4], [24]). Let $p \geq 2$ and $g_{1}, \ldots, g_{p}$ be $p$ holomorphic functions of $n$ variables defining a complete intersection in an open subset $V$ of $\mathbf{C}^{n}$. Then, for any test form $\varphi \in \mathcal{D}^{n, n-p+1}(V)$, the function of two complex variables

$$
\left(\lambda_{1}, \lambda_{2}\right) \mapsto \lambda_{2}^{p-1} \int_{V}\left|g_{1}\right|^{2 \lambda_{1}}\left|g_{2} \cdots g_{p}\right|^{2\left(\lambda_{2}-1\right)} \bigwedge_{k=2}^{p} \overline{d g_{k}} \wedge \varphi
$$

can be continued from $\left\{\operatorname{Re} \lambda_{1}>0, \operatorname{Re} \lambda_{2}>1\right\}$ as a meromorphic function in two complex variables. Moreover, this meromorphic continuation $\mathcal{M}_{g}(\lambda ; \varphi)$ can be written near the origin in $\mathbf{C}^{2}$ as

$$
\mathcal{M}_{g}(\lambda ; \varphi)=k_{0}(\lambda)+\lambda_{2}^{p-1}\left(\sum_{j=1}^{N} \frac{k_{j}(\lambda)}{\prod_{l=1}^{p-2}\left(\rho_{j l} \lambda_{1}+\sigma_{j l} \lambda_{2}\right)}\right)
$$

where $k_{0}, \ldots, k_{N}$ are holomorphic near the origin and the $\rho_{j l}$ (resp. $\sigma_{j l}$ ) are constants in $\mathbf{N}$ (resp. in $\left.\mathbf{N}^{*}\right)$.

Proof: The fact that the function (4.1) can be meromorphically continued to $\mathbf{C}^{2}$ and that its continuation has the form (4.2) near the origin is proved in details in [4, p. 70-72], from formula (3.34) up to formula $(3.40)$. To be more precise, in the mentioned reference, only the meromorphic continuation of

$$
\left(\lambda_{1}, \lambda_{2}\right) \mapsto \lambda_{1}^{p-1} \int_{V}\left|g_{1}\right|^{2 \lambda_{1}}\left|g_{2} \cdots g_{p}\right|^{2\left(\lambda_{2}-1\right)} \bigwedge_{k=2}^{p} \overline{d g_{k}} \wedge \bar{\partial} \psi
$$

(for some test form $\psi \in \mathcal{D}^{n, n-p}(V)$ ) was expressed in the form (4.2), but in fact the argument does not use at all the fact that the test form is the $\bar{\partial}$ of another one; therefore one could replace $\bar{\partial} \psi$ by any test form $\varphi$ and get the same result.

Lemma 4.2. Let $p \geq 2$ and $g_{1}, \ldots, g_{p}$ be $p$ holomorphic functions of $n$ variables defining a complete intersection in an open subset $V$ of $\mathbf{C}^{n}$. Let $g^{\prime}:=\left(g_{2}, \ldots, g_{p}\right)$. Then, for any test form $\varphi \in \mathcal{D}^{n, n-p+1}(V)$, the function of two complex variables

$$
\left(\lambda_{1}, \lambda_{2}\right) \mapsto \lambda_{2} \int_{V}\left|g_{1}\right|^{2 \lambda_{1}}\left\|g^{\prime}\right\|^{2 \lambda_{2}} \frac{\bigwedge_{k=2}^{p} \overline{d g_{k}} \wedge \varphi}{\left(\left|g_{2}\right|^{2}+\cdots+\left|g_{p}\right|^{2}\right)^{p-1}}
$$

can be continued from $\left\{\operatorname{Re} \lambda_{1}>0, \operatorname{Re} \lambda_{2}>1\right\}$ as a meromorphic function in two complex variables. Moreover, this meromorphic continuation $\mathcal{N}_{g}(\lambda ; \varphi)$ is holomorphic in a product of halfplanes $\left\{\operatorname{Re} \lambda_{1}>-\eta_{1}\right\} \times$ $\left\{\operatorname{Re} \lambda_{2}>-\eta_{2}\right\}$. 
Proof: Although implicitly given in [6] and [4, Section 5], the proof of this lemma is there more suggested than detailed, so we will write it out here completely following the basic ideas one can find for example in [7, proof of Proposition 9] or in Section 2 above. We first localize the problem and use a resolution of singularities $(\mathcal{X}, \Pi)$ for the hypersurface $\left\{g_{1}=g_{2}=\cdots=g_{p}=0\right\}$, so that, in the local chart $\omega$, we have in local coordinates $t_{1}, \ldots, t_{n}$,

$$
\Pi^{*} g_{k}(t)=u_{k}(t) t_{1}^{\alpha_{k 1}} \cdots t_{n}^{\alpha_{k n}}, \quad k=1, \ldots, n
$$

where the $u_{k}$ are invertible holomorphic functions on the local chart $\omega$ and the $\alpha_{k l}$ are positive integers. Then, as in Section 2, our analytic function $\lambda \mapsto \mathcal{N}(\lambda, \varphi)$ appears as the sum of terms

$$
\lambda_{2} \int_{\omega}\left|u_{1} m_{1}\right|^{\lambda_{1}} \frac{\left\|\Pi^{*} g^{\prime}\right\|^{2 \lambda_{2}}}{\left(\sum_{k=2}^{p}\left|u_{k} m_{k}\right|^{2}\right)^{p-1}} \bigwedge_{k=2}^{m} \overline{\partial u_{k} m_{k}} \wedge \rho \Pi^{*} \varphi
$$

where $\rho=\rho_{\omega}$ is the function associated to the local chart in some partition of unity subordonned to $\operatorname{Supp} \Pi^{*} \varphi$. In order to decompose an integral of the form (4.4), we use the toric variety $\widetilde{\mathcal{X}}$ (together with the projection proper map $\widetilde{\Pi}: \widetilde{\mathcal{X}} \mapsto \mathbf{C}^{n}$ ) corresponding to the closed convex hull (in $\mathbf{R}_{+}^{n}$ ) of

$$
\bigcup_{j=2}^{p}\left\{\left(\alpha_{j 1}, \ldots, \alpha_{j n}\right)+\mathbf{R}_{+}^{n}\right\}
$$

(see the proof of Lemma 2.2 in Section 2 above). This introduces a new decomposition of (4.4), with expressions of the form

$$
\lambda_{2} \int_{\varpi}\left|\widetilde{\Pi}^{*} u_{1} m_{1}\right|^{\lambda_{1}}\left|\mu_{j_{\varpi}}\right|^{2 \lambda_{2}} \frac{\bigwedge_{k=2}^{m} \overline{\partial v_{k} \mu_{\omega}} \wedge \xi_{\rho, \varpi} \widetilde{\Pi}^{*}\left(\rho \Pi^{*} \varphi\right)}{\left|\mu_{j_{\varpi}}\right|^{2(p-1)}\left(\sum_{k=2}^{m}\left|v_{k}\right|^{2}\right)^{p-1-\lambda_{2}}}
$$

where $\mu_{\varpi}$ is the distinguished monomial among the $\mu_{j}=\widetilde{\Pi}^{*} m_{j}, j=$ $2, \ldots, p$, the $v_{k}$ are the holomorphic functions defined as $\widetilde{\Pi}^{*}\left(u_{k} m_{k}\right)=$ $v_{k} \mu_{j_{\varpi}}$ (note that $v_{j_{\varpi}}$ is invertible in $\varpi$ ) and $\xi_{\rho, \varpi}$ comes from a partition of unity related to a covering of $\widetilde{\Pi}^{*}$ Supp $\rho$. Such an expression (4.5) can be written as

(4.6) $\lambda_{2} \sum_{\varpi} \int_{\varpi} \frac{\left|\widetilde{\Pi}^{*} u_{1} m_{1}\right|^{\lambda_{1}}\left|\mu_{j_{\varpi}}\right|^{2 \lambda_{2}}}{\mu_{j_{\varpi}}^{p-1}}\left(\tilde{\theta}_{\varpi, 1, \lambda_{2}}+\tilde{\theta}_{\varpi, 2, \lambda_{2}} \wedge \frac{\overline{d \mu_{j_{\varpi}}}}{\overline{\mu_{j_{\varpi}}}}\right) \wedge \widetilde{\Pi}^{*} \Pi^{*} \varphi$,

where $\tilde{\theta}_{\varpi, 1, \lambda_{2}}$ and $\theta_{\varpi, 2, \lambda_{2}}$ are smooth forms with respective types $(0, p)$ and $(0, p-1)$ depending holomorphically on the parameter $\lambda_{2}$. It is now immediate that the meromorphic continuation of $\lambda \mapsto \mathcal{N}(\lambda, \varphi)$ exists and that its polar set is included in a collection of hyperplanes $\beta_{0}+$ 
$\beta_{1} \lambda_{1}+\beta_{2} \lambda_{2}=0$, where $\beta_{0} \in \mathbf{N}$ and $\left(\beta_{1}, \beta_{2}\right) \in \mathbf{N}^{2} \backslash\{0\}$. In order to see that there are no polar hyperplanes with $\beta=0$ (and then we will be done), we need to look more carefully at the analytic continuation of expressions of the form

$$
\lambda_{2} \sum_{\varpi} \int_{\varpi} \frac{\left|\widetilde{\Pi}^{*} u_{1} m_{1}\right|^{\lambda_{1}}\left|\mu_{j_{\varpi}}\right|^{2 \lambda_{2}}}{\mu_{j_{\varpi}}^{p-1}} \tilde{\theta}_{\varpi, 2, \lambda_{2}} \wedge \frac{\overline{d \tau}}{\bar{\tau}} \wedge \widetilde{\Pi}^{*} \Pi^{*} \varphi,
$$

where $\tau$ is among the coordinates that divide the distinguished monomial $\mu_{j_{\varpi}}$. If $\tau$ does not appear in the decomposition of $\widetilde{\Pi}^{*} m_{1}$, then the integration by parts which is necessary in order to raise the singularity $\bar{\tau}$ implies just a division of the expression by $\lambda_{2}$ (instead of a combination of $\lambda_{1}$ and $\lambda_{2}$ as it should be if the hypothesis was not fulfilled). Since $\lambda_{2}$ was in the numerator, the new expression (after integration by parts with respect to $\bar{\tau}$ ) is holomorphic near the origin in $\mathbf{C}^{2}$. If $\tau$ appears in the decomposition of $\widetilde{\Pi}^{*} m_{1}$, it means that $\Pi \circ \widetilde{\Pi}\{\tau=0\}$ is included in the $n-p$ dimensional analytic set $\left\{g_{1}=\cdots=g_{p}=0\right\}$. This implies (for dimension reasons) that any antiholomorphic differential form $\widetilde{\Pi}^{*} \Pi^{*} \bigwedge_{j \in I} d \bar{\zeta}_{j}$, when $I \subset\{1, \ldots, n\}$, \#I=n-p+1, vanishes identically on $\tau=0$, which means that all its coefficients have $\bar{\tau}$ as a factor. In such a case,

$$
\lambda_{2} \sum_{\varpi} \int_{\varpi} \frac{\left|\widetilde{\Pi}^{*} u_{1} m_{1}\right|^{\lambda_{1}}\left|\mu_{j_{\varpi}}\right|^{2 \lambda_{2}}}{\mu_{j_{\varpi}}^{p-1}} \tilde{\theta}_{\varpi, 2, \lambda_{2}} \wedge \frac{\overline{d \tau}}{\bar{\tau}} \wedge \widetilde{\Pi}^{*} \Pi^{*} \varphi
$$

has only holomorphic singularities and therefore defines a holomorphic function of $\lambda$ at the origin. This completes the proof of Lemma 4.2.

Proof of Theorem 4.1: We now follow the proof given in [4, Section 5]. We will prove our theorem by induction on the number $n-m$. When $n=m$, we are in the discrete situation, so we know that the two currents $T_{f}$ and $\bigwedge_{k=1}^{m} \bar{\partial} \frac{1}{f_{k}}$ act in the same way on $(n, 0)$ test forms which are holomorphic near the set $\left\{f_{1}=\cdots=f_{n}=0\right\}$ and are both killed by all antiholomorphic functions which vanish on this set (see Theorem 1 for this property for the current $T_{f}$ and [14] for the analogous property for the Coleff-Herrera current). This implies that the action of the two currents coincide when $n-m=0$. We will assume from now on that the inductive hypothesis holds when $0 \leq \mathrm{nb}$ variables-nb functions $\leq k-1$ and we want to prove our result when we take $p-1$ functions $g_{2}, \ldots, g_{n}$ in $n$ variables, defining a complete intersection in $V \subset \mathbf{C}^{n}$, and such that $n-(p-1)=k$. There is no restriction if we suppose $V$ is a polydisk centered at the origin in $\mathbf{C}^{n}$ (since our problem is a local one). 
Let $\varphi$ be a $(n, n-p+1)$ test form in $V$ or the form $\varphi_{I} d \zeta \wedge d \bar{\zeta}_{J}$, $\# J=n-p+1$. It follows from the Noether Normalisation lemma (see for example [10]) that (with $V$ eventually restricted) one can find convenient coordinates - also denoted as $\left(\zeta_{1}, \ldots, \zeta_{n}\right)=\left(\zeta_{1}, \zeta^{\prime}\right)$ - so that $\varphi$ contains $d \overline{\zeta_{1}}, \operatorname{dim}\left\{\zeta_{1}=g_{2}=\cdots=g_{p}=0\right\}=n-p$ and, for any generic choice of $\zeta_{1}^{0}$,

$$
\operatorname{dim}_{\zeta^{\prime}}\left\{g_{2}\left(\zeta_{1}^{0}, \zeta^{\prime}\right)=\cdots=g_{p}\left(\zeta_{1}^{0}, \zeta^{\prime}\right)=0\right\} \leq n-p .
$$

We will work from now on in such a domain $V=D\left(0, r_{1}\right) \times D^{\prime}$, with these coordinates. Let $\varphi=d \overline{\zeta_{1}} \wedge d \zeta_{1} \wedge \psi$. Let us fix $\lambda_{1}=\lambda_{1}^{0}$ with $\operatorname{Re} \lambda_{1}^{0} \gg 0$ and consider $\lambda_{2}$ with $\operatorname{Re} \lambda_{2}>1$. It follows from Fubini's theorem that, if $g=\left(\zeta_{1}, g_{2}, \ldots, g_{p}\right)=\left(\zeta_{1}, g^{\prime}\right)$,

(4.8) $\mathcal{M}_{g}(\lambda, \varphi)$

$= \pm \frac{\lambda_{2}^{p-1}}{(p-1) !} \int_{D\left(0, r_{1}\right)}\left|\zeta_{1}\right|^{2 \lambda_{1}^{0}} d \overline{\zeta_{1}} \wedge d \zeta_{1} \wedge\left(\int_{D^{\prime}}\left|g_{2} \cdots g_{p}\right|^{2\left(\lambda_{2}-1\right)} \bigwedge_{k=2}^{p} \overline{\partial_{\zeta^{\prime}} g_{k}} \wedge \psi\right)$.

If $\operatorname{Re} \lambda_{2}>p-1$, we have also, for the same $\lambda_{2}^{0}$,

(4.9) $\mathcal{N}_{g}(\lambda, \varphi)$

$$
= \pm \lambda_{2} \int_{D\left(0, r_{1}\right)}\left|\zeta_{1}\right|^{2 \lambda_{1}^{0}} d \overline{\zeta_{1}} \wedge d \zeta_{1} \wedge\left(\int_{D^{\prime}}\left\|g^{\prime}\right\|^{2 \lambda_{2}} \frac{\bigwedge_{k=2}^{p} \overline{\partial_{\zeta^{\prime}} g_{k}} \wedge \psi}{\left(\left|g_{2}\right|^{2}+\cdots+\left|g_{p}\right|^{2}\right)^{p-1}}\right) \text {. }
$$

For $\zeta_{1}^{0}$ fixed (in a generic way), we know from Theorem 1.1 that the function $\mathcal{N}_{\zeta_{1}^{0}, g^{\prime}}$ of one variable $\lambda_{2}$, defined as

$$
\mathcal{N}_{\zeta_{1}^{0}, g^{\prime}}\left(\lambda_{2}, \psi\right):=\lambda_{2} \int_{D^{\prime}}\left\|g^{\prime}\left(\zeta_{1}^{0}, \zeta^{\prime}\right)\right\|^{2 \lambda_{2}} \frac{\bigwedge_{k=2}^{p} \overline{\partial_{\zeta^{\prime}} g_{k}\left(\zeta_{1}^{0}, \zeta^{\prime}\right)} \wedge \psi\left(\zeta_{1}^{0}, \zeta^{\prime}\right)}{\left(\sum_{k=2}^{p}\left|g_{k}\left(\zeta_{1}^{0}, \zeta^{\prime}\right)\right|^{2}\right)^{p-1}}
$$

(for Re $\lambda_{2}$ large enough) can be continued as a meromorphic function in $\mathbf{C}$ (with poles in $\{r \in \mathbf{Q}, r<0\}$.) Moreover, we have

$$
\mathcal{N}_{\zeta_{1}^{0}, g^{\prime}}(0, \psi)=\frac{(2 \pi i)^{p-1}(-1)^{\frac{(p-1)(p-2)}{2}}}{(p-1) !} T_{g^{\prime}\left(\zeta_{1}^{0}, \zeta^{\prime}\right),\{1, \ldots, p-1\}}\left(\psi\left(\zeta_{1}^{0}, \zeta^{\prime}\right)\right)
$$

(see Lemma 2.2). The same is true for the function of the complex variable $\lambda_{2}$ defined (also for $\operatorname{Re} \lambda_{2}$ large enough) by

$$
\begin{aligned}
& \mathcal{M}_{\zeta_{1}^{0}, g^{\prime}}\left(\lambda_{2}, \psi\right) \\
& \quad:=\lambda_{2}^{p-1} \int_{D^{\prime}}\left|g_{2} \cdots g_{p}\left(\zeta_{1}^{0}, \zeta^{\prime}\right)\right|^{2\left(\lambda_{2}-1\right)} \bigwedge_{k=2}^{p} \overline{\partial_{\zeta^{\prime}} g_{k}\left(\zeta_{1}^{0}, \zeta^{\prime}\right)} \wedge \psi\left(\zeta_{1}^{0}, \zeta^{\prime}\right)
\end{aligned}
$$


as known from [4]. Moreover, one can see in $[\mathbf{2 3}]$ or in $[\mathbf{1 2}$, Theorem 6.2 .1 , p. 107], that

$$
\mathcal{M}_{\zeta_{1}^{0}, g^{\prime}}(0, \psi)=(2 \pi i)^{p-1}(-1)^{\frac{(p-1)(p-2)}{2}}\left\langle\bigwedge_{k=2}^{n} \bar{\partial} \frac{1}{g_{k}\left(\zeta_{1}^{0}, \zeta^{\prime}\right)}, \psi\left(\zeta_{1}^{0}, \zeta^{\prime}\right)\right\rangle .
$$

Since we are dealing now with $p-1$ functions of $n-1$ variables, we have $n-1-(p-1)=n-p=k-1$, we can apply the inductive hypothesis and therefore obtain

$$
(p-1) ! \mathcal{N}_{\zeta_{1}^{0}, g^{\prime}}\left(0, \psi\left(\zeta_{1}^{0}, \zeta^{\prime}\right)\right)=\mathcal{M}_{\zeta_{1}^{0}, g^{\prime}}\left(0, \psi\left(\zeta_{1}^{0}, \zeta^{\prime}\right)\right)
$$

Our final step will be to check that for $\operatorname{Re} \lambda_{1}^{0}$ large enough, the analytic continuation (with respect to $\lambda_{2}$ ) commutes with the integration with respect to $\zeta_{1}$ in (4.8) and (4.9). We already know (by Lemma 4.1 and 4.2) that the functions

$$
\begin{aligned}
\lambda_{1} & \mapsto \mathcal{M}_{g}\left(\left(\lambda_{1}, 0\right), \varphi\right) \\
\lambda_{1} & \mapsto \mathcal{N}_{g}\left(\left(\lambda_{1}, 0\right), \varphi\right)
\end{aligned}
$$

are well defined as meromorphic functions and have no pole at the origin. Let us assume for the moment that analytic continuation (with respect to $\lambda_{2}$ and up to $\operatorname{Re} \lambda_{2}>0$ ) and integration with respect to $\zeta_{1}$ commute when $\operatorname{Re} \lambda_{1}^{0} \gg 0$. Then we will get that for $\operatorname{Re} \lambda_{1}^{0} \gg 0$, we have

$$
(p-1) ! \mathcal{N}_{g}\left(\left(\lambda_{1}^{0}, 0\right), \varphi\right)=\mathcal{N}_{g}\left(\left(\lambda_{1}^{0}, 0\right), \varphi\right) .
$$

Following the analytic continuation, this time with respect to $\lambda_{1}$, we get that

$$
(p-1) ! k_{0}(0,0)=\tilde{k}_{0}(0,0)
$$

which means, if we refer to Lemma 2.2 and to [23] or [12, Theorem 6.2.1, p. 107], that

$$
T_{g,\{1, \ldots, p-1\}}(\varphi)=\left\langle\bigwedge_{k=2}^{p} \bar{\partial} \frac{1}{g_{k}}, \varphi\right\rangle
$$

and concludes the proof of our inductive assumption when $n-p=k$.

It remains to explain why that analytic continuation (with respect to $\lambda_{2}$ up to $\left.\operatorname{Re} \lambda_{2}>-\eta\right)$ and integration with respect to $\zeta_{1}$ commute when $\operatorname{Re} \lambda_{1}^{0} \gg 0$ in (4.8) and (4.9). This was already explained in [4]. We will use here a different approach, based on the use of BernsteinSato relations instead of resolution of singularities. Such an approach seems more natural. It follows from Proposition 3 in $[8]$ that there exist analytic functions $h_{1}$ and $h_{2}$ in one complex variable $u$, defined in $D\left(0, r_{1}^{\prime}\right), r_{1}^{\prime} \leq r_{1}$, polynomials $b_{1}$ and $b_{2}$ in $\mathbf{C}\left[\nu, \lambda_{2}\right]$, differential operators 
$\mathcal{Q}_{1}\left(\nu, \lambda_{2} ; u, \zeta, \partial_{\zeta}\right)$ and $\mathcal{Q}_{2}\left(\nu, \lambda_{2} ; u, \zeta, \tilde{\zeta}, \partial_{\zeta}, \partial_{\tilde{\zeta}}\right)$ (polynomials in $\nu, \lambda_{2}, \partial$ and with analytic coefficients in $\zeta \in W \subset V$ for $\mathcal{Q}_{1}$, in $(\zeta, \tilde{\zeta}) \in W \times W$ for $\left.\mathcal{Q}_{2}\right)$, such that

$$
\begin{aligned}
h_{1}(u) b_{1}\left(\nu, \lambda_{2}\right)\left[\left(\zeta_{1}-u\right)^{\nu}\left(g_{2} \cdots g_{p}\right)^{\lambda_{2}}\right] & =\mathcal{Q}_{1}\left[\left(\zeta_{1}-u\right)^{\nu}\left(g_{2} \cdots g_{p}\right)^{\lambda_{2}+1}\right] \\
h_{2}(u) b_{2}\left(\nu, \lambda_{2}\right)\left[\left(\zeta_{1}-u\right)^{\nu} G(\zeta, \tilde{\zeta})^{\lambda_{2}}\right] & =\mathcal{Q}_{2}\left[\left(\zeta_{1}-u\right)^{\nu} G(\zeta, \tilde{\zeta})^{\lambda_{2}+1}\right]
\end{aligned}
$$

the above identities being understood as formal identities in $D\left(0, r_{1}^{\prime}\right) \times W$ or in $D\left(0, r_{1}^{\prime}\right) \times W \times W$ and

$$
G(\zeta, \tilde{\zeta}):=\sum_{k=2}^{p} g_{2}(\zeta) \overline{g_{2}(\overline{\tilde{\zeta}})}
$$

The second relation provides, if one substitutes $\tilde{\zeta}=\bar{\zeta}$ and repeats the reasoning in Lemma 2.4, the following formal identity in $D\left(0, r_{1}^{\prime}\right) \times V$

$$
h_{2}(u) b_{2}\left(\nu, \lambda_{2}\right)\left[\left(\zeta_{1}-u\right)^{\nu}\left\|g^{\prime}\right\|^{2 \lambda_{2}}\right]=\mathcal{Q}_{2}\left[\left(\zeta_{1}-u\right)^{\nu}\left\|g^{\prime}\right\|^{2\left(\lambda_{2}+1\right)}\right] .
$$

In order to express the analytic continuations of

$$
\begin{aligned}
& \lambda_{2} \mapsto \mathcal{M}_{\zeta_{1}^{0}, g^{\prime}}\left(\lambda_{2}, \psi\right) \\
& \lambda_{2} \mapsto \mathcal{N}_{\zeta_{1}^{0}, g^{\prime}}\left(\lambda_{2}, \psi\right)
\end{aligned}
$$

we just use the fact that for a $\mathcal{C}^{1}(n-1, n-1)$ form $\Phi$ with compact support in $V$

$$
\begin{aligned}
\int_{V \cap\left\{\zeta_{1}=\zeta_{1}^{0}\right\}} \Phi & =-\frac{1}{2 \pi i} \int_{V} \frac{d \zeta_{1} \wedge \bar{\partial} \Phi}{\zeta_{1}-\zeta_{1}^{0}} \\
& =-\frac{1}{2 \pi i}\left[\int_{V} \frac{\left|\zeta_{1}-\zeta_{1}^{0}\right|^{2 \nu}}{\zeta_{1}-\zeta_{1}^{0}} d \zeta_{1} \wedge \bar{\partial} \Phi\right]_{\nu=0} .
\end{aligned}
$$

We now express our two functions $\mathcal{M}_{g^{\prime}, \zeta_{1}^{0}}\left(\lambda_{2}, \psi\right)$ and $\mathcal{N}_{g^{\prime}, \zeta_{1}^{0}}\left(\lambda_{2}, \psi\right)$ (when $\left.\operatorname{Re} \lambda_{2} \gg 0\right)$ using formula (4.13) and then transform the two functions of $\left(\nu, \lambda_{2}\right)$ that appear with the help of formula (4.11) (to rewrite $\left.\mathcal{M}_{g^{\prime}, \zeta_{1}^{0}}\left(\lambda_{2}, \psi\right)\right)$ or $(4.12)$ (to rewrite $\mathcal{N}_{g^{\prime}, \zeta_{1}^{0}}\left(\lambda_{2}, \psi\right)$ ), with $u=\zeta_{1}^{0}$ (of course such $h_{1}(u) h_{2}(u) \neq 0$ ), as meromorphic expressions of $\lambda_{2}$ (in $\operatorname{Re} \lambda_{2}>-\eta$ ) with coefficients estimated in $C /\left|h_{1} h_{2}\left(\zeta_{1}^{0}\right)\right|^{M}$ for some very large $M$ and poles independent of $\zeta_{1}^{0}$. If we reduce $r_{1}$, then one can assume that on $D\left(0, r_{1}\right), h_{1} h_{2}(u)=\tilde{h}(u) u^{K}$, where $\tilde{h}$ is an invertible holomorphic function. If $\operatorname{Re} \lambda_{1}^{0} \gg 0$, all the coefficients in the meromorphic expressions are integrable (with respect to $\zeta_{1}^{0}$ ) when multiplied by $\left|\zeta_{1}^{0}\right|^{2 \lambda_{1}^{0}}$. This shows that the integration with respect to $\zeta_{1}^{0}$ and the analytic continuation with respect to $\lambda_{2}$ up to $\lambda_{2}=-\eta, \eta<0$, commute in this case. This concludes the proof of Theorem 4.1. 


\section{References}

[1] V. Arnold, A. Varchenko and S. Goussein-Sade, "Singularités des applications différentiables, volume II: monodromie et comportement asymptotique des intégrales", MIR, Moscou, 1986.

[2] D. Barlet and H.-M. Maire, Développements asymptotiques, transformation de Mellin complexe et intégration sur les fibres, in: "Séminaire d'Analyse P. Lelong-P. Dolbeault-H. Skoda, Années 1985/1986", Lecture Notes in Mathematics 1295, Springer-Verlag, Berlin, 1987, pp. 11-23.

[3] B. Berndtsson, A formula for interpolation and division in $\mathbf{C}^{n}$, Math. Ann. 263 (1983), 399-418.

[4] C. A. Berenstein, R. Gay, A. Vidras and A. Yger, "Residue currents and Bézout identities", Progress in Mathematics 114, Birkäuser Verlag, Basel, 1993.

[5] C. A. Berenstein, R. Gay and A. Yger, Analytic continuation of currents and division problems, Forum Math. 1 (1989), 15-51.

[6] C. A. Berenstein and A. YGer, D-modules and exponential polynomials, Compositio Math. 95 (1995), 131-181.

[7] C. A. Berenstein And A. Yger, Green currents and analytic continuation, J. Anal. Math. 75 (1998), 1-50.

[8] H. Biosca, Sur l'existence du polynôme de Bernstein générique à une application analytique, C. R. Acad. Sci. Paris Sér. I Math. 322 (1996), 659-662.

[9] C. A. Berenstein And A. Yger, Analytic residue theory in the non-complete intersection case, math e-prints, cv/9905051.

[10] J. E. BJÖRK, "Rings of differential operators", North-Holland Mathematical Library, North-Holland Publishing Co., Amsterdam, New York, 1979.

[11] J. E. BJÖRK, "Analytic D-modules and applications", Kluwer Academic Publisher, Dordrecht, 1993.

[12] J. E. BJöRK, Residue currents and $\mathcal{D}$-modules on complex manifolds, Preprint, Stockholm University, April 1996.

[13] B. Berndtsson and M. Passare, Integral formulas and an explicit version of the Fundamental Principle, J. Funct. Anal. 84 (1989), 358-372.

[14] N. Coleff And M. Herrera, "Les Courants Résiduels Associés à une Forme Méromorphe", Lecture Notes in Mathematics 633, Springer-Verlag, Berlin, 1978. 
[15] A. Dickenstein, R. Gay, C. Sessa and A. Yger, Analytic functionals annihilated by ideals, Manuscripta Math. 90 (1996), $175-223$.

[16] H. FedERER, "Geometric measure theory", Springer-Verlag, New York, 1969 .

[17] W. Fulton, "Introduction to toric varieties", Annals of Mathematical Studies 131, Princeton University Press, Princeton, NJ, 1993.

[18] I. S. Gradshteyn and M. Ryzhik, "Tables of Integrals, Series, and Products", Fourth edition, Academic Press, New York, 1980.

[19] P. Griffiths and J. Harris, "Principles of Algebraic Geometry", Pure and Applied Mathematics, Wiley \& Sons, New York, 1978.

[20] H. HironaKa, Resolution of singularities of an algebraic variety over a field of characteristic 0. I, II, Ann. of Math. (2) 79 (1964), 109-326.

[21] M. Kashiwara, $B$-functions and holonomic systems, Invent. Math. 38 (1976), 33-54.

[22] G. Kempf, D. Mumford, B. Saint-Donnat and F. Knudsen, "Toroidal embeddings. I", Lecture Notes in Math. 339, SpringerVerlag, New York, 1973.

[23] M. Passare, Courants méromorphes et égalité de la valeur principale et de la partie finie, in: "Séminaire d'Analyse P. LelongP. Dolbeault-H. Skoda, Années 1985/1986", Lecture Notes in Math. 1295, Springer-Verlag, New York, 1987, pp. 157-166.

[24] M. Passare And A. K. Tsikh, Residue integrals and their Mellin transforms, Canad. J. Math. 47 (1995), 1037-1050.

[25] M. Passare and A. K. Tsikh, Defining the residue of a complete intersection, in: "Complex Analysis, Harmonic analysis and Applications", Pitman Res. Notes Math. Ser. 347, Longman, Harlow, 1996, pp. 250-267.

[26] M. Passare, A. Tsikh and A. A. Cheshel, Multiple MellinBarnes integrals as periods of Calabi-Yau manifolds with several moduli, Teoret. Mat. Fiz. 109 (1996), 381-394.

[27] M. Passare, A. Tsikh and O. Zhdanov, A multidimensional Jordan residue lemma with an application to Mellin-Barnes integrals, in: "Contributions to Complex analysis and Analytic geometry" Aspects Math. E26, Vieweg, Braunschweig, 1994, pp. 233-241.

[28] C. SabBah, Proximité évanescente II. Equations fonctionnelles pour plusieurs fonctions analytiques, Compositio Math. 64 (1987), 213-241. 
[29] A. VidRas AND A. YGeR, On asymptotic approximations of the residual currents, Trans. Amer. Math. Soc. 350(10) (1998), $4105-4125$.

[30] A. VidRAS AND A. Yger, On some generalizations of Jacobi's Residue Formula, math. e-prints, ag/9905044.

Mikael Passare:

Matematiska institutionen

Stockholms Universitet

10691 Stockholm

Sweden

August Tsikh:

Otdelenie matematiki

Krasnoyarskskii Universitet

660062 Krasnoyarsk

Russia

Alain Yger:

Laboratoire de Mathématiques Pures

Université Bordeaux 1

33405 Talence

France

Primera versió rebuda el 23 de novembre de 1998, darrera versió rebuda el 2 de juliol de 1999. 